\title{
A Review of Anomalous Resistivity for the Ionosphere
}

\author{
K. Papadopoulos \\ Plasma Dynamics Branch, Plasma Physics Division \\ Naval Research Laboratory, Washington, D. C. 20375
}

\begin{abstract}
This is a general review of anomalous resistivity with emphasis on its applicability in space and more specifically on ionospheric plasmas. It is addressed to the general ionospheric community rather than the specialist. Therefore a substantial amount of rigor has been sacrificed in favor of simplified physical pictures. However, several prescriptions are presented, on the basis of which one can compute the anomalous resistivity resulting from each specific mechanism. Following a conceptual discussion of resistivity a general formalism is presented for its computation on the basis of the spectrum of electric fieid fluctuations. On the basis of this it is shown that stable nonthermal plasmas can at most enhance resistivity by a few percent. The same is true for collisionally driven instabilities. From the current-driven instabilities, only the ion acoustic instability can produce a steady state anomalous resistivity. The rest result in transient resistivity and the appearance of hot electron or ion spots. A more satisfying picture emerges when the low-frequency turbulence that produces resistivity is excited parametrically by a highfrequency instability. The case where such a driver arises from the interaction of precipitating electrons is discussed in detail. Finally, the relevance of the various resistivity mechanisms and their importance in ionospheric electron acceleration is discussed. Although a large number of physical notions are well understood, the efforts toward their incorporation into a gross modeling picture remain embarrassingly small.
\end{abstract}

\section{Preamble}

This review is written for nonspecialists. The primary goal of the paper is to provide a report on the nonlinear theory of conventional and unconventional forms of anomalous resistivity from a unified point of view and analyze the strengths and weaknesses of each mechanism as applied to ionospheric phenomena. In this connection we shall be concerned not only with the mechanisms per se but also with the resulting physical phenomena.

We do not intend to delve deeply into the mathematical and technical details, which at the level that we are concerned with serve only to obscure the basic physics. Accordingly, we provide the interested reader with an extensive list of original papers where most of the relevant details are discussed.

In the interest of brevity and clarity, we refer mainly to papers which we feel contain an integrated view of each particular physical mechanism, the advantage being that the interested reader can obtain a development more detailed than that offered here by consulting a few references rather than the entire literature. This is especially true for the case of ion sound instability, about which literally hundreds of papers have been written. Accordingly, we apologize to investigators who, although they might have pioneered the particular subject, are not quoted directly.

We should also emphasize that this review is not building theoretical models to compare and contrast with observations. Rather, it is emphasizing and illustrating the underlying physics by considering the simplest situations.

\section{INTRODUCTION}

The fundamental questions that we address in this review are the following: Given an electric field parallel to the magnetic field of the plasma, what is its relationship to the observed current, and what is the energy distribution of the current-carrying electrons? This is equivalent to determining the resistivity of the plasma and its velocity dependence. The main points considered include the following: What is classical resistivity? What is anomalous resistivity? How does one com-

Copyright ( 1977 by the American Geophysical Union.

Paper number 6R0695. pute it, and what is its relationship to the classical? What processes can produce it? What are the implications and the relevance of the various mechanisms with respect to ionospheric plasmas and substorm related phenomena? All these points are not independent, and so we will consider pieces of them concurrently and simultaneously.

The review divides itself into several parts. In section 3 we introduce the notions connected with the classical (collisional) resistivity of a plasma. In section 4 we introduce the concept of anomalous resistivity and outline the way in which it can be computed. In section 5 we review the current-driven instabilities and examine the type and values of anomalous resistivity that they produce. In section 6 we examine the possibility of anomalous resistivity when the bulk electron current is stable. In section 7 we examine some of the observable consequences of anomalous resistivitylike production of runaway electrons. Section 8 differs somewhat from the mainstream of the paper, which emphasizes the theoretical plasma physics notions involved in the problem of anomalous resistivity. For the sake of completeness, we list there some of the main ionospheric and magnetospheric observations related to anomalous resistance as well as some of the theoretical papers which clarify its role in the ionospheric-magnetospheric coupling. In the final section we examine the importance of the previously discussed processes with respect to the ionosphere and substorm observations. We should mention that the paper deals with anomalous resistivity due to collective plasma interactions and does not consider stationary laminar structures such as double layers [Block, 1975] or resistivity due to geometric effects such as neoclassical anomalous transport. For the convenience of the reader a list of symbols is presented at the end of the paper.

Consider now the first question of the introduction. What is resistivity?

\section{Classical Resistivity}

Under the influence of an electric field $E$ the electrons acquire a drift velocity with respect to the much heavier ions. If for simplicity we consider the ions as infinitely massive, the equation of motion of an electron is 


$$
d \mathrm{v} / d t=(e / m) \mathrm{E}-\nu \mathrm{v}
$$

In the absence of the frictional force due to collisions $(\nu=0)$ the electron velocity increases linearly with time $\mathrm{v}=(e \mathrm{E} / m) t$. When the electron suffers collisions with the heavier ions, it loses its directed velocity, so that its steady state velocity is given by

$$
\mathbf{v}=e \mathrm{E} / \boldsymbol{m v}
$$

On the basis of (2) we can find the required relationship between the electric field and the current as

$$
\mathrm{j} \equiv n e \mathrm{v}=n e^{2} \mathrm{E} / m \nu \equiv \sigma \mathrm{E}
$$

so that the conductivity is given by

$$
\sigma=n e^{2} / m \nu=\omega_{e}^{2} / 4 \pi \nu
$$

and the resistivity by

$$
\eta=m \nu / n e^{2}=4 \pi \nu / \omega_{e}^{2}
$$

Some of the complications arise when the exact nature of the electron-ion interaction, which is of the Coulomb type, is taken into consideration. The friction force as given in (1) implies that in every collision the particle loses momentum of the same order of magnitude as its initial momentum. In Coulomb collisions this occurs only for large angle collisions $(x \geq \pi / 2)$, where the potential energy $e^{2} / r$ is of the same order as the relative kinetic energy between the particles; i.e., $\frac{1}{m v^{2}} \approx$ $e^{2} / r$. The cross section $\sigma_{0}$ is then $\sigma_{0}=\pi r^{2}=4 \pi e^{4} / m^{2} v^{4}$, and the collision frequency is

$$
\nu=n \sigma v=4 \pi n e^{4} / m^{2} v^{2}
$$

In practice, owing to the long range of the Coulomb force, small angle collisions are more frequent, and the cumulative effect of many small angle deflections turns out to be larger by a factor $\ln \Lambda$, so that (6) becomes

$$
\nu=\left(4 \pi n e^{4} / m^{2} v^{a}\right) \ln \Lambda
$$

Since $\Lambda=4 \pi n \lambda_{D}{ }^{3}, \ln \Lambda$ is between 10 and 20 for most plasmas of interest. When the drift velocity of the electrons $V_{D}$ is smaller than their thermal velocity $V_{e}\left(V_{D} \leqslant V_{e}\right)$, the relative velocity between electrons and ions $v$, which is the quantity entering (7), is $v \approx V_{e}$, so that from (5) and (7) we recover the well-known Spitzer formula for the classical resistivity of a plasma as

$$
\eta \approx\left(\pi e^{2} m^{1 / 2} / T_{e}^{3 / 2}\right) \ln \Lambda
$$

It is interesting to note that under the above condition $\left(V_{D} \leq\right.$ $V_{e}$ ) the collision frequency of (7) becomes

$$
\nu \approx f \omega_{e}(\ln \Lambda / \Lambda)
$$

Equation (9) indicates the connection of the collisional time scale due to the discreteness of particles to the collective time scale $\omega_{e}$ in terms of the plasma parameter $\Lambda$.

However, some serious complications appear when the drift velocity exceeds the thermal velocity of the plasma $\left(V_{D}>V_{e}\right)$. When $V_{D}<V_{e}$, the friction force $F=\nu m V_{D} \sim V_{D} / V_{e}^{*}$ increases with the drift velocity, and a steady state like the one given by (2) can be achieved. In the opposite regime $\left(V_{e}<V_{D}\right)$, $F \approx \nu m V_{D} \sim 1 / V_{D}{ }^{2}$, i.e., decreases with directed velocity. This behavior is graphically shown in Figure 1. Under such conditions a steady state cannot be achieved, and the electron population runs away. We can therefore determine the maximum permissible electric field in a plasma under steady state condi- tions. This field is called the runaway or Dreicer field $E_{D}$, and its value as found from (2) and (7) with $v \approx V_{e}$ is given by

$$
E_{D}=\left(e / \lambda_{D}^{2}\right) \ln \Lambda
$$

This conclusion, however, appears to contradict the experimental observations which do not indicate runaway behavior for electric fields substantially larger than $E_{D}$. This fact can be explained only if the resistivity was substantially larger than that computed previously, and it has led to an extensive research for the causes of the larger resistivity, commonly called anomalous resistivity. This will be the subject of the subsequent sections.

There is one more interesting phenomenon that can be presented within the same simplified framework of the resistivity. As was shown above (Figure 1), the friction force is a function of velocity. The calculation of the runaway field $E_{D}$ was done within a fluid framework which characterizes the electrons by two variables $V_{D}$ and $V_{e}$. However, since in reality the electrons are distributed around $V_{e}$, there will be a number of fast particles that will run away even for $E<E_{D}$. Given the electron distribution function, we can compute the number of runaways. The velocity $V_{r}$ above which runaway occurs for a given $E<E_{D}$ as found from (6) and (10) is

$$
V_{r}=\left(E_{D} / E\right)^{1 / 2} V_{e}
$$

For a Maxwellian distribution a rough estimate of the fraction of the runaway particles is given by

$$
\begin{aligned}
\frac{n_{r}}{n}=\frac{1}{2} \exp \left\{-\frac{1}{2}\left[\left(\frac{E_{D}}{E}\right)^{1 / 2}-\right.\right. & \left.\left.\frac{E}{E_{D}}\right]^{2}\right\} \\
& \approx \frac{1}{2} \exp \left(-\frac{1}{2} \frac{E_{D}}{E}\right)
\end{aligned}
$$

As we will see in a later section, wuch an effect can have a profound influence on the plasma resistivity because the runaways can form electron beams which can produce plasma waves, thereby influencing the plasma conductivity.

\section{General Formulation of Resistivity}

We commented in the previous section on the problems encountered when the electric field exceeds the runaway field $E_{D}$. The resolution of this problem lies in the fact that when the electron velocity relative to the ions exceeds some threshold value whose upper value is $V_{e}$, plasma collective effects become important. The plasma becomes unstable to the generation of electrostatic waves which grow at the expense of electron kinetic energy. The electrons subsequently collide not only with the fields of individual ions but with these growing self-consistent fields. The scattering of the electrons by these fields shows up as an additional momentum loss. Physically, the instability spatially correlates the ions by bunching them so that the colliding electrons see an effective charge to mass ratio much larger than $e^{2} / M$.

The question that we will try to answer in this section is this: Given the state of turbulence of a plasma, what are the resistivity and the heating rates of the electrons and ions? The turbulence of course is excited by some instability, and its characteristic spectrum will depend on the particular instability mechanism. Determination of the stabilized turbulence spectrum for the various relevant mechanisms will be the subject of the next section. In this section we assume that the turbulent spectrum is given, and we determine the resistivity as a function of the correlation functions. Of the various approaches developed in the last few years [Hasegawa, 1974, 
1975; Tsytovich, 1972; Sagdeev and Galeev, 1969; Gary and Paul, 1971] we follow the one suggested by Tange and Ichimaru [1974] because of its generality.

We again follow a fluidlike description of the electrons and ions, which implies that our variables are the relative drift velocity $V_{D}$ and the electron and ion temperatures $T_{e}, T_{l}$ (or equivalently $V_{e}, V_{l}$ ). The transport equations for these quantities, which can be found by taking the first and second moment of the kinetic equations in velocity space, involve only the density correlation functions among the species. For a uniform system with temporal variation the two time correlation functions, $\left\langle n_{l}(\mathbf{r}, t) n_{j}\left(\mathbf{r}^{\prime}, t^{\prime}\right)\right\rangle$, depend not only on $\mathbf{r}-\mathbf{r}^{\prime}$ and $t$ $-t^{\prime}$ but also on the average time $\left(t+t^{\prime}\right) / 2$. By assuming the latter dependence to be much slower than $t-t^{\prime}$ we can carry out the Fourier transformation of the correlation functions with respect to $r-r^{\prime}$ and $t-t^{\prime}$ as

$$
\begin{aligned}
\left\langle n_{t}(\mathrm{r}, t) n_{j}\left(\mathrm{r}^{\prime}, t^{\prime}\right)\right\rangle & \\
=\exp & \int \frac{d \mathbf{k}}{(2 \pi)^{3}} \int d \omega S_{i j}\left(\mathrm{k}, \omega ; \frac{t+t^{\prime}}{2}\right) \\
& {\left[i \mathrm{k} \cdot\left(\mathrm{r}-\mathrm{r}^{\prime}\right)-i \omega\left(t-t^{\prime}\right)\right] }
\end{aligned}
$$

It can be shown that the transport equations depend only on the values of the spectral functions $S_{i}(\mathbf{k}, \omega ; t)$. We present here the results and refer the interested reader to Tange and Ichimaru [1974] for a detailed derivation. The momentum equation is given by

$$
\frac{d}{d t} \mathbf{V}_{D}-\frac{e}{m} \mathbf{E}=\frac{e^{2}}{n m} \int \frac{d \mathbf{k}}{(2 \pi)^{3}} \int d \omega \frac{4 \pi \mathbf{k}}{k^{2}} \operatorname{lm} S_{e l}(\mathbf{k}, \omega ; t)
$$

while the energy equations for the electrons and ions are

$$
\begin{aligned}
& \frac{d}{d t} T_{e}=4 \pi e^{2} \int \frac{d \mathbf{k}}{(2 \pi)^{3}} \int d \omega \frac{\left(\omega-\mathbf{k} \cdot \mathbf{V}_{D}\right)}{k^{2}} \operatorname{Im} S_{e l}(\mathbf{k}, \omega ; t) \\
& \frac{d}{d t} T_{\imath}=4 \pi e^{2} \int \frac{d \mathbf{k}}{(2 \pi)^{3}} \int d \omega \frac{\omega}{k^{2}} \operatorname{Im} S_{e t}(\mathbf{k}, \omega ; t)
\end{aligned}
$$

A word of caution with respect to (15) and (16) seems appropriate. First, they are correct in the ion reference frame; namely, $V_{D}$ is the relative velocity of the electrons with respect to the ions. Second, the meaning of the temperatures $T_{e}, T_{1}$ is generalized to include sloshing energy due to the interaction of the particles with the waves. That is, the quantity $\sum, W_{t \jmath}$, where

$$
W_{i j}=\frac{q_{\imath} q_{j}}{2} \int \frac{d \mathbf{k}}{(2 \pi)^{3}} \int d \omega \frac{4 \pi}{k^{2}} S_{i j}(\mathbf{k}, \omega ; t)
$$

is added to the thermal energy. However, since even in cases of strong turbulence these contributions are small, in most practical cases, (15) and (16) can be regarded as describing the heating rates of the electrons and ions. One additional energy equation which includes the electric field and current is

$$
\frac{d}{d t}\left[\sum_{j} T_{j}+\frac{1}{2} n m V_{D}^{2}\right]=\mathrm{j} \cdot \mathbf{E}
$$

Equations (14)-(17) are applicable to both quiescent and turbulent plasmas. With the knowledge of the spectral functions Im $S_{e t}(\mathbf{k}, \omega ; t)$ the rates of momentum and energy transfers can be determined. Since often the quantities measured experimentally or computed are the electric field fluctuations rather than the correlation functions $S_{i}(\mathbf{k}, \omega ; t)$, it is useful and convenient to rewrite (14)-(16) in terms of the electric field fluctuations of

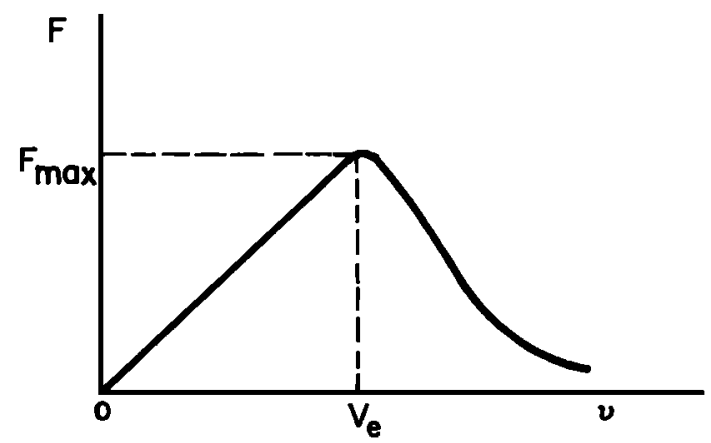

Fig. 1. Frictional force $F$ between electrons and ions as a function of their relative velocity $v$.

a turbulent plasma. The relationship between the electric field fluctuations $\mathbf{E}(\mathbf{k}, \omega)$ and the induced density fluctuations $n_{j}(\mathbf{k}$, $\omega)$ is given by [Sitenko, 1967]

$$
q_{\jmath} n_{f}(\mathbf{k}, \omega ; t)=-\chi,(\mathbf{k}, \omega ; t)(1 / 4 \pi) \mathbf{k} \cdot \mathrm{E}(\mathbf{k}, \omega ; t)
$$

$\chi,(k, \omega ; t)$ is the polarizability as determined by the exact particle orbits in the presence of the turbulent fields. We can then express $S_{e l}(\mathbf{k}, \omega ; t)$ as

$$
\left.S_{e l}(\mathbf{k}, \omega ; t)=-\left[k^{2} /(4 \pi)^{2} e^{2}\right]\left\langle E^{2}\right| \mathbf{k}, \omega ; t\right) \chi_{e}(\mathbf{k}, \omega ; t) \chi_{i}(\mathbf{k}, \omega ; t)
$$

If a stationary state exists, then in its time scale

$$
\epsilon(\mathbf{k}, \omega ; t)=1+\sum_{j} x_{j}(\mathbf{k}, \omega ; t)=0
$$

at any time, and (19) gives the required relationship

$\left.\operatorname{Im} S_{e t}(\mathbf{k}, \omega ; t)=\left[k^{2} /(4 \pi)^{2} e^{2}\right]\left\langle E^{2}\right| \mathbf{k}, \omega ; t\right) \operatorname{Im} \chi_{e}(\mathbf{k}, \omega ; t)$

From (14)-(16) and (20) we find the basic transport equations for a plasma in a quiescent or turbulent stationary state. They are given by

$$
\begin{aligned}
\frac{d}{d t} \mathbf{V}_{D} & -\frac{e}{m} \mathbf{E} \\
& =\frac{1}{4 \pi m n} \int \frac{d \mathbf{k}}{(2 \pi)^{3}} \int d \omega \mathbf{k}\left\langle E^{2} \mid \mathbf{k}, \omega ; t\right\rangle \operatorname{Im} \chi_{e}(\mathbf{k}, \omega ; t)
\end{aligned}
$$

$\frac{d}{d t} T_{e}=\frac{1}{4 \pi} \int \frac{d \mathbf{k}}{(2 \pi)^{3}} \int d \omega\left(\omega-\mathbf{k} \cdot \mathrm{V}_{D}\right)\left\langle E^{2} \mid \mathbf{k}, \omega ; t\right\rangle \operatorname{Im} \chi_{e}(\mathbf{k}, \omega ; t)$

$\frac{d}{d t} T_{t}=\frac{1}{4 \pi} \int \frac{d \mathbf{k}}{(2 \pi)^{3}} \int d \omega \omega\left(E^{2}\left|\mathbf{k}, \omega ; t^{\prime}\right\rangle \operatorname{Im} \chi_{\mathrm{e}}(\mathbf{k}, \omega ; t)\right.$

Equations (14)-(16) or (21)-(23) form a basic set of equations necessary for the computation of anomalous resistivity and the heating rates. $A$ basic ingredient for the anomalous processes is the existence of a spectrum of electrostatic waves $\left\langle E^{2} \mid \mathbf{k}, \omega ; t\right\rangle$ at all times. These processes disappear where the field spectrum vanishes. Therefore if we expect a steady state resistivity, the agent exciting these waves (instability, external sources, etc.) should operate during the entire time of interest. If the excited waves are due to an initially unstable configuration which becomes linearly stable owing to the anomalous friction (equation (21)) or heating (equations (21) and (22)), they can produce only transient resistivity, and it is inappropriate to speak of a steady state anomalous resistivity. We will see several such examples in the following section. For the cases that a steady state can be established, it is convenient to define 
an anomalous collision frequency $\nu^{*}$ on the basis of (21). By defining $\nu^{*}$ in an equivalent fashion with (2) we find

$$
\begin{aligned}
\nu^{*}=\frac{e\left(E \cdot V_{D}\right)}{m V_{D}^{2}}= & \frac{e^{2}}{m^{2} V_{D}^{2} \omega_{e}^{2}} \int \frac{d \mathbf{k}}{(2 \pi)^{3}} \\
& \cdot \int d \omega\left(\mathbf{k} \cdot V_{D}\right)\left(E^{2} \mid \mathbf{k}, \omega\right) \operatorname{Im} \chi_{e}(\mathbf{k}, \omega)
\end{aligned}
$$

It is convenient for our future use to express (24) in the dimensionless form given below:

$$
\begin{aligned}
& \nu^{*}=\frac{V_{e}}{V_{D}} \frac{1}{8 \pi n T_{e}} \int \frac{d \mathbf{k}}{(2 \pi)^{3}} \int d \omega \frac{\left(\mathbf{k} \cdot \mathbf{V}_{D}\right)}{\left(k V_{D}\right)} \\
& \cdot\left(k \lambda_{D}\right)\left(E^{2} \mid \mathbf{k}, \omega\right) \operatorname{Im} \chi_{e}(\mathbf{k}, \omega)
\end{aligned}
$$

We should caution the reader that the word steady state is used here in a time average sense rather than a strictly time independent one.

From the above discussion we see that the necessary inputs for computation of the resistivity are experimental or theoretical knowledge of the spectrum $\left(E^{2} \mid \mathbf{k}, \omega\right)$ at stabilization and of the corresponding nonlinear susceptibility $\chi_{e}(k, \omega)$. The necessary condition for anomalous resistivity is the presence of a quasi-stationary spectrum $\left\langle E^{2} \mid \mathbf{k}, \omega\right\rangle$ such that $\nu^{*}$ computed by (25) is larger than the relevant binary collision frequency (electron-ion or electron-neutral). Several physical notions can be clarified if we examine how the above rigorous results compare with the ones derived by the simplified physical analysis of section 3 for the case of stable plasmas. In this case the values of $\operatorname{Im} S_{e l}(\mathbf{k}, \omega)$ or equivalently of $\left\langle E^{2} \mid \mathbf{k}, \omega\right\rangle$ can be computed by using the method of superposition of dressed particles [Rostoker. 1961; Papadopoulos, 1970]; they are given by

$q_{e} q_{i} \operatorname{Im} S_{e l}(\mathbf{k}, \omega)=\left[q_{e}{ }^{2} S_{e}^{(0)}(\mathbf{k}, \omega) \operatorname{Im} \chi_{\imath}{ }^{0}(\mathbf{k}, \omega)\right.$

$$
\left.-q_{i}{ }^{2} S_{i}^{(0)}(k, \omega) \operatorname{Im} \chi_{e}^{0}(k, \omega)\right] /\left.\epsilon^{0}(k, \omega)\right|^{2}
$$

The superscript implies that the relevant functions have been computed on the basis of straight orbits. Thus

$$
S_{j}^{(0)}(\mathrm{k}, u)=\frac{1}{2 \pi} \int d \mathrm{v} f(\mathrm{v}) \int_{-\infty}^{\infty} d t \exp (i \mathrm{k} \cdot \mathrm{v} t-i \omega t)
$$

For the case of Maxwellian electrons drifting with velocity $\mathbf{V}_{D}$ through ions the polarizabilities are given by

$$
\begin{aligned}
\chi_{e}^{0} & =\frac{1}{k^{2} \lambda_{D}{ }^{2}} Z\left(\frac{\omega-\mathbf{k} \cdot V_{D}}{k V_{e}}\right) \\
\chi_{l}^{0} & =\frac{1}{k^{2} \lambda_{D}{ }^{2}} \frac{T_{e}}{T_{i}} Z\left(\frac{\omega}{k V_{l}}\right) \\
\epsilon^{0}(k, \omega) & =1+\chi_{e}^{0}+\chi_{i}^{0}
\end{aligned}
$$

The function $Z(y)$ is the usual plasma dispersion function defined by

$$
\begin{aligned}
Z(y)=1-y \exp \left(-\frac{y^{2}}{2}\right) & \int_{0}^{y} d y \exp \left(\frac{y^{2}}{2}\right) \\
& +t\left(\frac{\pi}{2}\right)^{1 / 2} y \exp \left(-\frac{y^{2}}{2}\right)
\end{aligned}
$$

On the basis of (14) and (24)-(30) and the fact that $\nu=$ $e E \cdot V_{D} / m V_{D}^{2}$ we find that

$$
\nu=\frac{e^{2}}{(2 \pi)^{1 / 2} V_{e} n \lambda_{D}^{2} m} \int_{0}^{\infty} \frac{d k}{k} \int_{-1}^{+1} d y y
$$

$$
\int_{-\infty}^{\infty} d \omega \frac{S_{l}^{(0)}(\mathbf{k}, \omega)}{|\epsilon(\mathbf{k}, \omega)|^{2}}\left[\left(\frac{T_{e}}{T_{l}}-1\right) \frac{\omega}{k V_{D}}+y\right]
$$

Equation (31) offers an opportunity to examine the discrete versus the collective part of resistivity. The key factor is the $1 /|\epsilon(\mathbf{k}, \omega)|^{2}$ dependence. As long as $|\epsilon(\mathbf{k}, \omega)| \neq 0$, one can carry out the integrations in a straightforward manner. However, when $|\epsilon(k, \omega)|=0$, in which case collective modes appear, a resonance integration across the pole in the denominator becomes necessary. We can therefore write as usual [Montgomery and Tidman, 1964]

$$
\frac{1}{|\epsilon(\mathbf{k}, \omega)|^{2}}=\mathbf{P} \frac{1}{|\epsilon(\mathbf{k}, \omega)|^{2}}+\pi \sum_{i}\left|\frac{\partial R_{e} \epsilon}{\partial \omega} \operatorname{Im} \epsilon\right| \delta\left(\omega \pm \omega_{J}\right)
$$

where $\omega_{j}$ is the frequency of the collective modes. For an unmagnetized plasma we can approximate

$$
\mathbf{P} \frac{1}{|\epsilon(\mathbf{k}, \omega)|^{2}}=1+\frac{1}{\left(k \lambda_{D}\right)^{2}}\left[1+\frac{T_{e}}{T_{l}} Z\left(\frac{\omega}{k V_{l}}\right)\right]
$$

Integrating (31) with the aid of (32) between the inverse of the distance of closest approach $k_{0}=T_{e} / e^{2}$ and $k_{D}=1 / \lambda_{D}$, we find the collision frequency due to single-particle encounters [Spitzer, 1956; Tange and Ichimaru, 1974] as

$$
\begin{aligned}
\nu=\frac{1}{3} \frac{\omega_{e}}{\Lambda}\left[\ln \Lambda-\left(1+\frac{T_{l}}{T_{e}}\right)\right. & \ln \left(1+\frac{T_{e}}{T_{l}}\right) \\
& \left.+\left(2+\frac{T_{l}}{T_{e}}\right) \frac{1}{\Lambda^{2}}+\cdots\right]
\end{aligned}
$$

which is similar to the result found in section 3 .

The remaining contribution due to the second term of $(32)$ is due to the collective interactions $\left(k \lambda_{D}<1\right)$. It is interesting to examine whether these contributions can produce any significant enhancements for the case of a stable plasma with a drift velocity approaching the threshold velocity for instability. The general problem is very complex, although the physical interpretation of the results is rather simple. We present here a simplified derivation and refer the interested reader to Ichimaru [1973] and Papadopoulos [1965]. We examine the case of marginally stable ion acoustic waves with $T_{e} \gg T_{i}$. The dispersion relation is given by

$$
\begin{aligned}
& \epsilon(\mathbf{k}, \omega) \approx\left(1+\frac{1}{\left(k \lambda_{D}\right)^{2}}\right)\left(1-\frac{\omega_{k}^{2}}{\omega^{2}}\right) \\
&+i\left(\frac{\pi}{2}\right)^{1 / 2} \frac{1}{k^{2} \lambda_{D}^{2}} \frac{V_{D}(k)-V_{D}}{V_{e}}
\end{aligned}
$$

The value $V_{D}(k)$ is the velocity for marginal stability of the mode number $k$. It is an increasing function of $k$ which indicates that large wavelength waves $(k \rightarrow 0)$ will become unstable first. Its approximate value is given by [Papadopoulos, 1965]

$$
V_{D}(k)=C_{8}\left[1+\frac{1}{2}\left(k \lambda_{D}\right)^{2}+\cdots\right]
$$

Notice that as $V_{D}$ approaches $V_{D}(k)$, the imaginary part of $\epsilon(\mathbf{k}$, $\omega)$ tends to vanish, which implies an infinite lifetime for the mode (i.e., instability onset). Using (31), (32a), (34a), and (34b) we find after some complex algebra that the collision frequency $\nu_{\text {col }}$ due to the collective part is given by

$$
\nu_{\text {col }} \approx \frac{1}{f}\left(\omega_{e} / \Lambda\right)\left(k_{c} \lambda_{D}\right)^{s}
$$

where $k_{c}$ is the critical wavelength for the particular drift velocity. The ratio $\nu_{\text {col }} / \nu \approx\left(k_{c} \lambda_{D}\right)^{\mathrm{s}}(1 / \ln \Lambda)$, and since $k_{c} \lambda_{D} \ll$ 
1 and $\ln \Lambda \simeq 6-10, \nu_{\mathrm{col}} / \nu<<1$. Namely, the contribution of marginally stable modes to the plasma resistivity is negligible, despite the fact that the spectrum of the ion density fluctuations is dramatically increased. The physical explanation of this can be traced to the fact that for any stable plasma the total electrostatic fluctuation energy

$$
\int d \mathbf{k} \int d \omega\left(E^{2} \mid \mathbf{k}, \omega\right)
$$

is bounded by $T_{e}$. Sharpening the ion wave feature by varying $T_{e} / T_{l}$ and $V_{D} / V_{e}$ results in more concentration of this energy in the ion wave feature, but an integration over frequency and wave number can only give the net area under the entire spectrum which is a strictly bounded quantity. Only an unstable situation or an external source can remove this bound and produce anomalous resistivity [Hirshfield, 1973].

In this section we derived the relevant transport equations in the presence of an electric field (equations (14)-(16) or equations (21)-(23)) and the steady state anomalous collision frequency (equations (24) and (25)) as a function of the turbulence spectrum and the particle distribution function. It was shown that for stable plasmas we can recover the results of section 3 and that critical fluctuations cannot provide more than a few percent increase in the plasma resistance.

\section{Anomalous Resistivity Due to Current-Driven INSTABILITIES}

It was shown in the previous section that a stable plasma, even if it approaches from below the limit of being marginally unstable, cannot have any significant enhancement of its resistivity. In view of this we turn our attention here to the possibility of anomalous resistivity in an unstable plasma. From the form of (21)-(25) we have seen that in order for the electrons to experience friction the phase velocity $\left(v_{\mathrm{ph}}=\omega / k\right)$ of the waves should be small $\left(\omega / k \ll V_{e}, V_{D}\right)$. This implies that lowfrequency waves $\left(\omega \ll \omega_{e}\right)$ are the ones that will produce anomalous resistance. Since such waves can be easily produced by currents, a voluminous amount of work has been performed with respect to current-driven instabilities [Kindel and Kennel, 1971; Hasegawa, 1974]. We examine below the main linear and nonlinear features of the various current-driven instabilities and assess the potential for anomalous resistivity in the ionospheric plasma. We list in Table 1 the main currentdriven instabilities and their typical linear characteristics and proceed to examine each one separately.

\section{Buneman Instability}

This is one of the oldest known instabilities. It occurs when there is an electron and ion distribution function in the form of two delta functions displaced relative to each other by the drift velocity $V_{D}$ (Figure 2). It is essentially independent of the ratio $T_{e} / T_{l}$, and its threshold velocity is given by $V_{D} \geq 1.7\left(V_{e}+V_{l}\right)$.

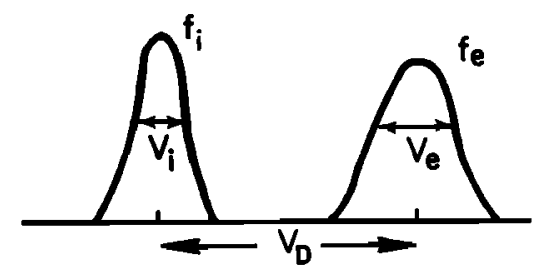

Fig. 2. Electron and ion velocity distributions for the case of Buneman instability.

It leads to excitation of longitudinal electrostatic oscillations predominantly in the direction of the drift. By neglecting electron thermal effects the dispersion relation in the electron reference frame is given by [Buneman, 1959; Davidson et al., 1970; Kindel and Kennel, 1971]

$$
\frac{\omega_{t}^{2}}{\left(\omega-\mathbf{k} \cdot V_{D}\right)^{2}}+\frac{\omega_{e}^{2}}{\omega^{2}}=1
$$

The maximum growth occurs where $\mathbf{k} \cdot \mathrm{V}_{D}=\omega_{e}$, and with $\omega=$ $\omega_{r}+i \gamma$ we find

$$
\omega_{r}=\omega_{e} \quad \gamma=\left[(3)^{1 / 2} / 2\right](m / 2 M)^{1 / 3} \omega_{e}
$$

The nonlinear theory of the instability was studied first by Davidson et al. [1970] using one-dimensional particle simulation methods. It was four $d$ that it saturates by electron trapping which brings the system to marginal stability by heating the electrons to the point where $V_{e} \approx V_{D}$. A very small amount of energy $\left(\sim(m / M)^{1 / 3}\right)$ goes to the ions in the form of a small tail. These studies were performed by imparting an initial drift between electrons and ions. Of more relevance to the problem of anomalous resistivity were studies performed when the plasma was submitted to a constant electric $E_{0}$ [Boris et al., 1970; Morse and Nielson, 1971; Biskamp and Chodura, 1973; Orens and Dawson, 1974]. On the basis of the nonlinear theory of the Buneman instability one would expect the following picture. The electrons are initially freely accelerated till their drift velocity exceeds their thermal velocity. At this point the Buneman instability starts operating. The fast momentum loss of the electrons keeps the drift velocity constant and heats the electrons to the point where the thermal speed $V_{e}$ becomes larger than $V_{D}$. This cycle then repeats itself. The numerical simulations of Boris et al. [1970] confirmed this picture as shown in Figure 3a. There is no constant anomalous resistivity, only a time dependent one, composed of almost square pulses (Figure $3 b$ ). The electrons are therefore running away but at half their free acceleration rate, while the rest of the energy goes into electron heating. The main features of this situation are [Biskamp and Chodura, 1973] as follows: (1) The energy supplied to the system is used predominantly to accelerate and heat the electrons. (2) The system seems.to evolve in a self-similar way: $V_{D} \sim t, V_{e} \sim t,\left(E^{2}\right\rangle / 8 \pi n T_{e} \approx$ const, and $\langle k\rangle^{-1}$

TABLE 1. Characteristics of Important Current-Driven Instabilities

\begin{tabular}{lcccc}
$\begin{array}{c}\text { Type of } \\
\text { Instability }\end{array}$ & $\begin{array}{c}\text { Threshold } \\
\text { and } \\
\text { Conditions }\end{array}$ & $\begin{array}{c}\text { Frequency } \\
\text { (in the Ion } \\
\text { Reference Frame) }\end{array}$ & $\begin{array}{c}\text { Growth } \\
\text { Rate }\end{array}$ & Comments \\
\hline Buneman & $V_{D}>V_{e}$ & $\sim(m / M)^{1 / s} \omega_{e}$ & $\sim(m / M)^{1 / 9} \omega_{e}$ & $\begin{array}{c}\text { Independent of the } \\
\text { ratio } T_{e} / T_{l}\end{array}$ \\
Ion acoustic & $V_{D}>C_{a}$ & $\leq \omega_{l}$ & $<\omega_{l}\left(V_{D} / V_{e}\right)$ & $\begin{array}{c}\text { Depends critically } \\
\text { on the ratio } T_{e} / T_{l}\end{array}$ \\
Ion cyclotron & $V_{D}>C_{*}$ & $\approx \Omega_{t}$ & $\approx \Omega_{l}\left(V_{D} / V_{e}\right)$ & $\begin{array}{c}\text { Occurs even for } \\
T_{e} / T_{t} \approx 1\end{array}$ \\
\hline
\end{tabular}




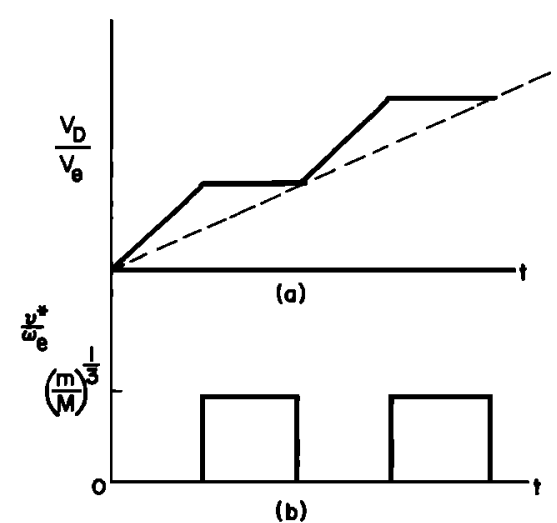

Fig. 3. Schematic of the evolution of an electron-ion plasma in the presence of a strong electric field. (a) The system evolves in a selfsimilar fashion with $V_{D} \sim V_{e} \sim t$. The electrons are running away at half the free acceleration rate. (b) There is no steady state anomalous resistivity. The collision frequency is time dependent and in the form of square pulses.

$\sim t$. In particular, the electron distribution function remains self-similar. (3) $V_{D} \approx V_{e}$ to a high degree of accuracy apart from oscillations that they both perform about each other.

It is therefore inappropriate to attrivute steady state anomalous resistivity effects to a Buneman instability. One of the puzzling features of the above observations is the fact that even in cases where the electron acceleration rate is much smaller than the growth rate of the ion acoustic instability and $T_{e} / T_{t} \gg 1$, no ion acoustic instability was observed in the simulations. The explanation of this was that a small ion tail was formed at the acoustic speed during the Buneman stage, as can be seen from the simulations of Davidson et al. [1970]. Ion Landau damping in the tail stabilized the ion acoustic modes despite the fact that $V_{D} \gg C_{S}$ and $T_{e} / T_{\imath} \gg 1$. All the above studies were one-dimensional. Two-dimensional simulations performed by Lampe et al. [1974] revealed that the ion tail cannot stabilize the off-angle modes of the ion acoustic instability and the Buneman instability evolves to an ion sound instability if its threshold, which will be discussed below, is exceeded. We should note that for the Buneman instability, one-dimensional behavior is expected for $\Omega_{e}>\omega_{e}$.

In summary, we conclude that Buneman instability cannot provide steady state anomalous resistivity and prevent runaway in one-dimensional systems $\left(\Omega_{e}>\omega_{e}\right)$, while in multidimensional systems the steady state resistivity will be due to the ion acoustic instability. This is discussed next.

\section{Ion Acoustic Instability}

This is probably the most controversial instability with respect to its nonlinear behavior. Its linear theory is rather simple. In some sense it is a continuation of the Buneman instability when the drift velocity $V_{D}$ becomes smaller than the electron thermal velocity. In this case it becomes important to consider kinetic effects, since the conditions for hydrodynamic instability, i.e., $V_{l}<(m / M)^{1 / 2} V_{D}$ and $V_{e}<V_{D}$, are violated. A general form of instabilities that arise in an electron-ion plasma in which there is a relative drift is shown in Figure 4. Instability depends essentially on the ratio $T_{e} / T_{i}$. For $T_{e} / T_{i} \approx$ 1 , only the hydrodynamic instability (i.e., Buneman) arises and requires $V_{D} \gtrsim V_{e}$.

For displaced Maxwellians the dispersion relation is given by [Mikhalouskii, 1974; Kindel and Kennel, 1971; Ossakow. 1968] $\epsilon(\mathbf{k}, \omega)=1+\frac{1}{k^{2} \lambda_{D}^{2}}\left[Z\left(\frac{\omega-\mathbf{k} \cdot V_{D}}{k V_{e}}\right)+\frac{T_{e}}{T_{i}} Z\left(\frac{\omega}{k V_{i}}\right)\right]$

In the frequency domain where

$$
\left(\frac{T_{l}}{M}\right)^{1 / 2}<\frac{\omega_{r}}{|\mathbf{k}|}<\left(\frac{T_{e}}{M}\right)^{1 / 2}+V_{D}
$$

the dispersion relation (38) becomes

$$
\begin{aligned}
1+\frac{1}{k^{2} \lambda_{D}^{2}} & \left(1+\frac{i(\pi)^{1 / 2}\left(\omega-\mathbf{k} \cdot V_{D}\right)}{|k| V_{e}}\right)-\frac{\omega_{t}^{2}}{\omega^{2}}\left(1+3 \frac{k^{2} T_{i}}{M \omega^{2}}\right) \\
& +\frac{i(\pi)^{1 / 2}}{k^{2} \lambda_{D}^{2}} \frac{T_{e}}{T_{i}} \frac{\omega}{|k| V_{t}} \exp \left(-\frac{\omega^{2}}{k^{2} V_{l}^{2}}\right)=0
\end{aligned}
$$

From (38c) it follows that the plasma is unstable if $V_{D}>V_{\mathrm{cr}}$, where

$$
\begin{array}{r}
V_{\mathrm{cr}}=\left(\frac{T_{e}}{M}\right)^{1 / 2}\left(1+k^{2} \lambda_{D}{ }^{2}\right)^{-1 / 2}\left[1+\left(\frac{T_{e}}{T_{l}}\right)^{3 / 2}\left(\frac{M}{m}\right)^{1 / 2}\right. \\
\left.\cdot \exp \left(-\frac{3}{2}-\frac{T_{e}}{2 T_{l}\left(1+k^{2} \lambda_{D}{ }^{2}\right)}\right)\right]
\end{array}
$$

For $T_{e} / T_{l}$ large (i.e., $\left.T_{e} / T_{l}>\ln \left[\left(T_{e} / T_{l}\right)^{\mathrm{s}} M / m\right]\right)$ the minimum of $V_{\text {cr }}$ occurs at $\left(k \lambda_{D}\right)^{2} \geq 1$. In this case, ion waves are excited with frequency

$$
\omega_{r}^{2}=\omega_{i}^{2}\left(1+3 k^{2} \lambda_{D i}^{2}\right)
$$

at which case instability occurs if

$$
V_{D}>\left(\frac{T_{e}}{M}\right)^{1 / 2} \ln ^{1 / 2}\left[\left(\frac{T_{e}}{T_{\imath}}\right)^{3} \frac{M}{m}\right]
$$

For smaller $T_{e} / T_{i}$ ratios the first waves to become unstable have $k^{2} \lambda_{D}^{2} \ll 1$. In this stage the instability is called ion acoustic, has frequency

$$
\omega_{r}^{2}=\left(k^{2} T_{e} / M\right)\left[1+\left(3 T_{i} / T_{e}\right)\right]
$$

and occurs when

$$
V_{D}>\left(\frac{T_{e}}{M}\right)^{1 / 2}\left[1+\left(\frac{T_{e}}{T_{\ell}}\right)^{1 / 2}\left(\frac{M}{m}\right)^{1 / 2} \exp \left(-\frac{3}{2}-\frac{T_{e}}{2 T_{\imath}}\right)\right]
$$

The growth rate in both cases is given by

$$
\begin{aligned}
\gamma_{k}=-\left(\frac{\pi}{8}\right)^{1 / 2} \frac{\omega_{r}}{\left(k \lambda_{D}\right)^{2}}\left(\frac{\omega_{r}}{\omega_{i}}\right)^{2} \\
\cdot\left[\frac{\omega_{r}-\mathbf{k} \cdot V_{D}}{k V_{e}}+\frac{T_{i}}{T_{e}} \exp \left(-\frac{\omega_{r}^{2}}{2 k^{2} V_{e}^{2}}\right)\right]
\end{aligned}
$$

In Figure 5 we present a graph of the $V_{\text {cr }}$ as a function of $T_{e} / T_{l}$ as computed by Jackson [1960] for hydrogen. For $T_{e} \approx$ $T_{l}$ the value of $V_{\mathrm{er}} \approx 0.9 V_{e}$ as found by Buneman. We can see that for $V_{D} / V_{e} \ll 1$, which is typical of space plasmas, substantial temperature ratios are required for instability $\left(T_{e} / T_{i}\right.$ $\approx 8-10$ ).

Before closing on the linear theory we mention a hot ion kinetic instability situated in the upper right-hand corner of Figure 4. It occurs for $V_{D}>V_{e}$ and hot ions $\left(T_{t} / T_{e}>\right.$ $\left.(M / m)^{1 / 2}\right)$. In view of this strong restriction we will not discuss it any further.

While the linear theory of the instability is well under control, the nonlinear theory has been the subject of large con- 


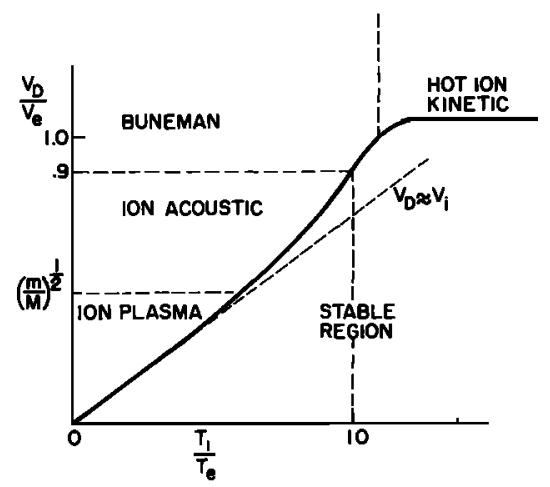

Fig. 4. Instability diagram for the case of relative electron-ion drift with no magnetic field showing the type of unstable waves.

troversy. From (21)-(25) we see that in order to compute the anomalous resistivity and the heating rates, one requires the spectrum $\left\langle E^{2} \mid \mathbf{k}, \omega_{r}\right\rangle$ of stabilization and the nonlinear susceptibility Im $\chi_{e}$. A common characteristic of the presently acceptable nonlinear theories is connected with the fact that the stabilization occurs owing to effects of the turbulence on the ions. The electron trajectories are not significantly altered by the presence of the acoustic turbulence. We can therefore use in all the above theories for the $\operatorname{Im} \chi_{e}$ its linear value

$$
\operatorname{Im} \chi_{e}(\omega, \mathbf{k})=\left(\frac{\pi}{2}\right)^{1 / 2} \frac{1}{\left(k \lambda_{D}\right)^{2}} \frac{\omega-\mathbf{k} \cdot V_{D}}{k V_{e}}
$$

The difference among the various theories is in the way they compute the stabilized spectrum. A detailed discussion lies beyond the scope of the present review. We limit ourselves here to a brief description of the basic nonlinear mechanisms that can provide a stabilized spectrum of turbulence.

Weak turbulence theories. The first of the weak turbulence theories was advanced by Kadomtsev [1965]. The stationary turbulent spectrum is computed by balancing the linear (or quasi-linear) growth of the instability against the nonlinear scattering of the unstable waves on the plasma ions (nonlinear Landau damping). Since $V_{t}{ }^{2} / C_{s}{ }^{2}=T_{t} / T_{e} \ll 1$, the plasma kinetic equations can be expanded in this small parameter. To the lowest order the resulting equation is given by

$$
\begin{aligned}
& \frac{\partial}{\partial t}\left\langle E^{2} \mid \mathbf{k}\right\rangle=\frac{\omega_{k}^{3}}{k^{2} C_{s}{ }^{2}}\left(\frac{\pi}{2}\right)^{1 / 2}\left(\frac{m}{M}\right)^{1 / 2}\left(\frac{V_{D}}{C_{B}} \cos \theta-\frac{\omega_{k}}{k C_{s}}\right)\left\langle E \mid \mathbf{k}^{2}\right\rangle \\
& +\frac{\omega_{i}{ }^{2} \omega_{k}{ }^{3}}{n M^{2} k^{2}} \int d \mathbf{k}^{\prime} \frac{\left(\mathbf{k} \cdot \mathbf{k}^{\prime}\right)^{2}\left(\mathbf{k} \times \mathbf{k}^{\prime}\right)^{2}}{\omega_{k}{ }^{3} \omega_{k^{\prime}}{ }^{2}\left|\mathbf{k}-\mathbf{k}^{\prime}\right|^{2} k^{\prime 2}} \int d \mathbf{v} \delta\left[\omega_{k}-\omega_{k^{\prime}}\right.
\end{aligned}
$$$$
\left.-\left(\mathbf{k}-\mathbf{k}^{\prime}\right) \cdot \mathbf{v}\right]\left(\mathbf{k}-\mathbf{k}^{\prime}\right) \cdot \frac{\partial f_{t}}{\partial \mathbf{v}}\left\langle E^{2} \mid \mathbf{k}\right\rangle\left\langle E \mid \mathbf{k}^{\prime 2}\right\rangle
$$

where $\omega_{k}$ and $\omega_{k}$, are given by (39). The second term on the right-hand side represents the nonlinear Landau damping. Equation (43), being an integral equation, does not have a stable stationary solution. However, there are solutions oscillating periodically between the Cerenkov angle $\theta_{0}$, defined by $\cos \theta_{0}=C_{s} / V_{D}$, and the current line $\theta=0$. When we average over these oscillations and integrate (43) over the angle $\theta$, the left-hand side essentially vanishes. In the limit of $\theta_{0}$ $\ll 1$ and $k \lambda_{D} \ll 1$ the equation can be solved [Kadomisev, 1965; Sagdeev and Galeev, 1969]. The result is the so-called Kadomtsev spectrum:

$$
\begin{gathered}
W(\mathbf{k})=\frac{\left\langle E^{2} \mid \mathbf{k}\right\rangle}{8 \pi n T_{e}}=\frac{1}{4}\left(\frac{\pi}{2}\right)^{1 / 2}\left(\frac{m}{M}\right)^{1 / 2}\left(\frac{V_{D}}{C_{\theta}}\langle\cos \theta\rangle-1\right) \\
\cdot \frac{T_{e}}{T_{l}} \frac{T_{e}}{4 \pi e^{2}\left(\theta^{2}\right)} \frac{1}{k^{6}} \ln \frac{1}{k \lambda_{D}}
\end{gathered}
$$

This result is correct for $k \lambda_{D} \ll 1$, since the strong linear ion Landau damping contribution at $k \lambda_{D} \approx 1$, which has been neglected, will essentially cut off the spectrum. The total wave energy obtained by integrating over $k$ is

$$
W=\frac{\left\langle E^{2}\right\rangle}{8 \pi n T_{e}}=\frac{1}{16 \pi}\left(\frac{\pi}{2}\right)^{1 / 2} \frac{1}{\left\langle\theta^{2}\right\rangle} \frac{T_{e}}{T_{i}}\left(\frac{m}{M}\right)^{1 / 2}\left(\frac{V_{D}}{C_{s}}\langle\cos \theta\rangle-1\right)
$$

From (25) and (43) we find the so-called Sagdeev resistivity given by

$$
\nu^{*}=10^{-2}\left(T_{e} / T_{l}\right)\left(V_{D} / V_{e}\right) \omega_{e}
$$

The numerical factor $10^{-2}$ emerges during the calculations and is $1 / 32 \pi$ [Biskamp, 1972].

A variation of this theory is the renormalized weak turbulence theory advanced by Tsytovich [1971, 1972]. He noted that although the spectrum of ion sound oscillations is a nondecay one, in the region of low wave numbers, the threewave resonance interaction condition can be met if the theory is renormalized to consider the effect of the short correlation time in the presence of finite amplitude ion waves (i.e., nonlinear line broadening). He then utilized this to allow for threeway resonance in the kinetic equation for waves as a nonlinear term to achieve saturation. With the exception of the $T_{i} / T_{e}$ dependence the stabilized spectrum is similar to the Kadomtsev spectrum, while the resistivity has a somewhat larger value than the one given by Kadomtsev. This theory has been criticized [Sagdeev, 1974] as lacking a region of applicability because it applies to wave numbers $k \lambda_{D} \ll 1$, while the dominant spectrum of ion sound is around $k \lambda_{D} \approx 1$.

We should mention that several of the difficulties and divergences of the weak turbulence theory have recently been resolved by the work of Choi and Horton [1974] and Horton et al. [1976].

Orbit modification theory. This theory advanced by Dupree [1966] and Weinstock [1967] replaces the unperturbed orbits of the linear theory by a more exact statistical orbit in the presence of finite amplitude turbulence. Its dominant effect is that it broadens the resonance region for wave-particle interactions. The details of the calculation can be found in papers by Sleeper and Weinstock [1972], Sleeper et al. [1973], and Wesson and Sykes [1973]. The essential physical notion is that the broadening of ion Landau resonance due to perturbed ion orbit effects in the turbulent plasma can increase the wave damping to the point that it balances the growth. The system then remains in a nonlinear marginally stable state, and the required resonance broadening fixes the turbulence level. At saturation,

$$
\operatorname{Im} \chi_{i}^{\mathrm{NL}}(\mathbf{k}, \omega)=-\operatorname{Im} \chi_{e}^{0}(\mathbf{k}, \omega)
$$

$\operatorname{Im} \chi_{i}{ }^{N L}(k, \omega)$ is similar to $\operatorname{Im} \chi_{l}^{0}(k, \omega)$ if we replace $V_{l}^{2} \rightarrow V_{l}^{2}$ $+\Delta V_{t k}^{2}$, where

$$
\Delta V_{i k}=\left(\frac{4(2)^{1 / 2} \pi^{3 / 2} e^{2}}{3 M^{2}} \frac{k_{i} k_{j}}{k^{3}} \sum_{k^{\prime}} \frac{k_{l}^{\prime} k_{j}^{\prime}}{k^{\prime 3}} \frac{W_{k^{\prime}}}{\Delta V_{l k^{\prime}}}\right)^{1 / 3}
$$


is the resonance broadening induced by $W_{k^{\prime}}$. From (40) and (47) we can determine the stabilized turbulence level. This has been done numerically by Sleeper et al. [1973], and it depends on the ratios $T_{e} / T_{t}$ and $V_{D} / V_{e}$. The results are shown in Figure 6. The anomalous collision frequency is given by $\nu^{*}=$ $\omega_{e}\left(W / n T_{e}\right)$ with $W$ taken from Figure 6.

There are several other theories proposed in order to explain some of the detailed observations, like ion tails [Caponi and Davidson, 1974; Manheimer and Flynn, 1974]. Since the values of $\nu^{*}$ found are similar to the above, we do not discuss them any further but refer the interested reader to Orens and Dawson [1974], who compare the various theories with particle simulations. We should mention also some attempts at self-similar solutions of the ion acoustic instability in the spirit of the ones given for the Buneman instability, which, however, presented more problems than the ones they solved [Sagdeev, 1974].

Before concluding this section we should note that with respect to the ion sound instability the condition $T_{l} / T_{e}<1$ should always be satisfied, because otherwise the ions will acquire larger temperatures than the electrons leading to violation of condition $(41 b)$ and premature damping of the waves. In this case the instability will result in a hot ion spot rather than steady state resistivity. On the basis of (22)-(23) we find that for steady state anomalous resistivity

$$
\frac{T_{l}}{\dot{T}_{e}}=\frac{T_{e}}{T_{i}} \frac{C_{s}}{V_{D}} \frac{1}{\left[1-\left(C_{s} / V_{D}\right)\right]}<1
$$

In summary, we can state that the ion acoustic instability can produce a steady state anomalous resistivity if (48) is satisfied. The value $\nu^{*}$ will be given as $\nu^{*} / \omega_{e}=W / n T_{e}$ with $W$ taken either from Figure 6, (45), or any other theory or experimental value.

\section{Ion Cyclotron Instability}

For drift velocities not very large $\left(V_{D} \ll V_{e}\right)$ the ion acoustic instability considered above occurs only in a strongly nonisothermal plasma $\left(T_{e} / T_{l} \gg 1\right)$. For $T_{e} \sim T_{l}$, ion acoustic waves do not occur because of the strong ion Landau damping. However, the presence of a magnetic field increases the number of plasma modes that can be excited by eliminating the ion Landau damping transverse to the field. This makes possible the excitation of ion cyclotron waves in the case of low electron drifts. The linear dispersion relation has been detailed by Drummond and Rosenbluth [1962]. When we use displaced Maxwellians (Figure 4) and restrict ourselves to the first cyclotron harmonic (e.g., $\omega<2 \Omega_{\imath}$ ), we find [Benford, 1976]

$$
\epsilon(k, \omega)=1+\frac{1}{\left(k \lambda_{D}\right)^{2}}\left[1+i \pi^{1 / 2} \frac{\left(\omega-k_{\|} V_{D}\right)}{k_{\| \mid} V_{e}}\right]
$$

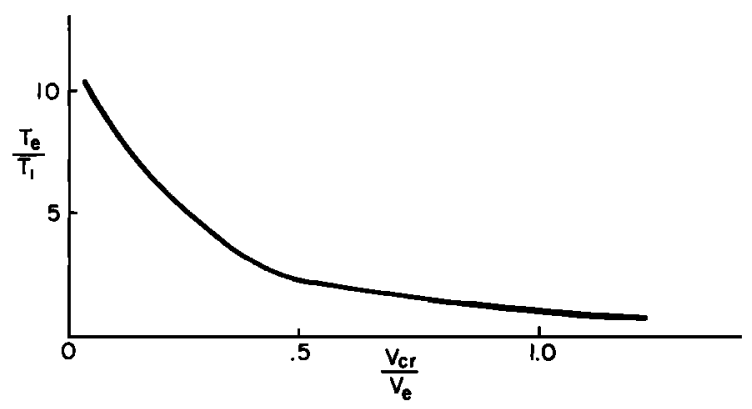

Fig. 5. Critical relative drift velocity as a function of $T_{e} / T_{t}$.

$$
-\frac{\beta}{\left(k \lambda_{D}\right)^{2}} \frac{T_{l}}{T_{e}}\left\{\frac{\omega}{\omega-\Omega_{i}}+i \pi^{1 / 2} \frac{\omega}{k_{\|} V_{l}} \exp \left[-\frac{1}{2}\left(\frac{\omega-\Omega_{l}}{k_{\|} V_{l}}\right)^{2}\right]\right\}
$$

where

$$
\beta=\left(T_{e} / T_{i}\right)^{-s} I_{1}(s) \quad s=\left(k_{\perp} V_{l} / \Omega_{i}\right)^{2}
$$

From (49) one can determine the forms for linear frequency $\omega_{r}$ near the first cyclotron harmonic and the growth rate as

$$
\begin{aligned}
& \omega_{r}=(1+\beta) \Omega_{l} \\
& \gamma_{k}=\gamma_{e}+\gamma_{\imath}=\left(\frac{\pi}{2}\right)^{1 / 2} \beta\left\{\Omega_{\imath}\left(\frac{V_{D}}{V_{e}}-\frac{\Omega_{l}}{k_{\|} V_{e}}\right)\right. \\
&-\left.\omega\left(\frac{\omega-\Omega_{l}}{k_{\|} V_{l}}\right) \exp \left[-\frac{1}{2}\left(\frac{\omega-\Omega_{l}}{k_{\|} V_{l}}\right)^{2}\right]\right\}
\end{aligned}
$$

From these equations we find that the threshold condition for the ion cyclotron instability is given by

$$
V_{D}>u_{c}=15\left(T_{l} / T_{e}\right) V_{l}=15\left(T_{l} / T_{e}\right)^{9 / 2}(m / M)^{1 / 2} V_{e}
$$

which for a hydrogen plasma reduces to $V_{D} / V_{e}>\left(T_{i} / T_{e}\right)^{3 / 2}$. The qualitative dependence of the frequency and the growth rate on $s=\left(k_{\perp} V_{t} / \Omega_{l}\right)^{2}$ is shown in Figure 7 . This is actually correct if $V_{D} / u_{c} \gg 1$. When $V_{D} \rightarrow u_{c}$, the band of unstable waves becomes substantially narrower.

Within the framework of a one-dimensional quasi-linear theory, Petviashvili [1963] showed that the appearance of a plateau in the electron distribution function will stabilize the ion cyclotron waves. One can then compute the resulting effective collision frequency self-consistently with the result

$$
\nu^{*} \approx \nu_{e l}\left[1+\left(V_{D} / V_{e}\right)\right]
$$

namely, an increase of only a few percent, which is essentially insignificant. Similar properties are expected from other lowfrequency $\left(\omega \ll \Omega_{e}\right)$ kinetic instabilities, such as the universal and the Alfvén wave instability.

It was subsequently argued [Vedenov, 1968; Kadomstev, 1965; Dum and Dupree, 1970] that collisions, spatial effects, and the presence of longitudinal laminar electric fields will prevent plateau formation and that saturation will occur at a higher level by some other mechanism, thus opening the possibility for larger resistivities. More recently, Palmadesso et al. [1974] reexamined the problem under the assumption that plateau formation is inhibited in some way. It was shown that in this case the dominant nonlinear stabilization mechanism was ion resonance broadening, which results in a wave energy level given by

$$
W=\frac{k_{\perp}^{2}}{2 \pi e^{2}} T_{i}^{2}\left(\frac{\omega_{r}-\Omega_{i}}{\Omega_{\iota}}\right)^{2}
$$

However, it was shown that although $W$ as given by (54) can be large, it cannot provide a steady state anomalous resistivity. The reason is that according to (22)-(23) the ion heating rate $\dot{T}_{i}$ greatly exceeds the electron heating rate $\dot{T}_{e}$ (e.g., $\dot{T}_{l} / \dot{T}_{e}>>$ $1)$. It was actually calculated that $T_{l} \approx \frac{1}{2} \gamma_{l \mathrm{c}} T_{l}$. On the basis of this result and the fact that the critical velocity $u_{c}$ as given by (52) is proportional to $T_{t}^{3 / 2}$, we expect that the ion heating will increase the critical velocity so as to bring the system to stability, at which point the instability will shut off and the turbulence and heating rates will be greatly reduced. Experimental evidence in support of this view has been provided by Kelley et al. [1975b]. In conclusion, we expect the ion cylotron 
instability to produce a local hot ion spot and shut off. With respect to anomalous resistivity we expect a situation similar to that in the Buneman instability, namely, a temporary increase but not any significant steady state enhancement.

\section{Collisional Instabilities}

In the previous parts of this section we considered currentdriven instabilities for a collisionless plasma. It is well known [Mikhalouskii, 1974] that for weakly ionized plasmas, currentdriven collisional (or dissipative) instabilities can be excited [Self, 1970; Coppi and Mazzucato, 1971]. In this case, electronneutral or electron-ion collisions drive the instability, while ion-neutral collisions can damp them. There are experimental observations of large $\delta n / n \approx 0.9$ due to collisional ion acoustic waves [Karatzas et al., 1975]. Such collisional modes are expected to be present below $1000-\mathrm{km}$ altitudes in the ionosphere [Hudson, 1975; Mozer, 1975]. It is therefore important to determine whether they can produce anomalous resistance. As we noted in section 3 , the presence of a linear instability does not warrant any significant additional resistivity, and a nonlinear analysis should be performed before any reliable conclusions can be extracted. However, for the particular case of collisional waves, some general upper bounds can be derived. The anomalous collision frequency is given by (24). Let us take, for example, the collisional ion waves. In this case [ $\mathrm{Ka}$ ratzas et al., 1975],

$$
\operatorname{Im} \chi_{e}^{0}(\mathbf{k}, \omega)=\frac{1}{\left(k \lambda_{D}\right)^{2}} \frac{\nu_{0}}{k V_{e}} \frac{\omega-\mathbf{k} \cdot \mathbf{V}_{D}}{k V_{e}}
$$

where $\nu_{0}$ is the relevant collison frequency $(e-n$ or $e-i)$. Using (24) and (55), we find

$$
\frac{\nu^{*}}{\omega_{e}} \approx \frac{1}{\left(k \lambda_{D}\right)^{2}} \frac{\nu_{0}}{\omega_{e}} \frac{W}{n T_{e}}
$$

At this point we notice that for ion waves

$$
\left(\frac{\delta n}{n}\right)^{2}=\frac{1}{2} \cdot \frac{1}{\left(k \lambda_{D}\right)^{2}} \frac{W}{n T_{e}}
$$

so that $(56 a)$ becomes

$$
\frac{\nu^{*}}{\omega_{e}}=\frac{1}{2} \frac{\nu_{0}}{\omega_{e}}\left(\frac{\delta n}{n}\right)^{2}
$$

Therefore even for $\delta n / n \approx 1, \nu^{*}<\nu_{0}$. Similar considerations can be shown to apply to any collisional instability. The physical reason can be easily traced if we notice that these instabilities have very long wavelengths $\left(k \lambda_{D} \sim \nu_{0} / \omega_{e}\right)$, and therefore the electric fields associated with these are very small and not very effective in deflecting the electrons.

Before closing we should note that some of the conclusions of Ronglien and Self [1971] with respect to collisional ion waves with very small drifts were shown to be in error by McBride and Chu [1972] due to their neglect of the temperature equation.

\section{Anomalous Resistivity in Plasmas Stable to CURRENT-Driven INSTABILITIES}

As was seen in the previous section, one of the basic problems in determining a current-driven instability mechanism that can provide anomalous resistance in the ionospheric plasma is the fact that unrealistically high currents are required to excite ion sound under conditions where $T_{e} \approx T_{l}$, and even then, we might end up with a hot spot and only

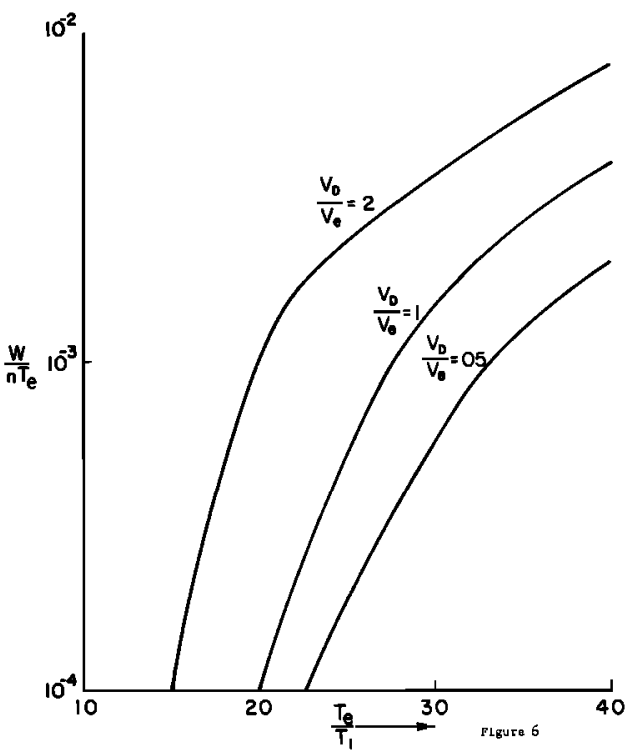

Fig. 6. Values of $W / n T_{e}$ at stabilization for the ion acoustic instability according to resonance broadening theory [Sleeper et al. 1973]. Notice strong dependence on $T_{e} / T_{t}$ and $V_{D} / V_{e}$ near threshold and weaker dependence away $(M / m=1836)$.

transient resistivity. In view of this, Papadopoulos and Coffey $[1974 a, b]$ examined the possibility that anomalous resistivity can be generated in a plasma stable to current-driven instabilities. As was shown in section 4 (equations (21)-(25)), the key to obtaining anomalous resistance is the presence of a nonthermal spectrum of low-frequency density fluctuations which can efficiently scatter the drifting electrons. For the processes discussed in section 5 this spectrum was driven by the currents themselves. However, as Papadopoulos and Coffey $[1974 a, b]$ noted, if such a spectrum could be generated and sustained by some process independent of the electron current, the drifting electrons will see an increased friction [Dupree, 1970; Hui and Dupree, 1975]. At this point they turned their attention to how such a spectrum could be created in the ionospheric plasma. They noted that a large amount of the available free energy resides in the fast (5-20 keV) precipitating electron streams which are unstable to high-frequency waves (plasma waves, whistler waves, etc.). However, since these waves have phase velocities much larger than the drifting electrons, typically around the velocity of the precipitating particles, they cannot provide any enhanced resistance. At this

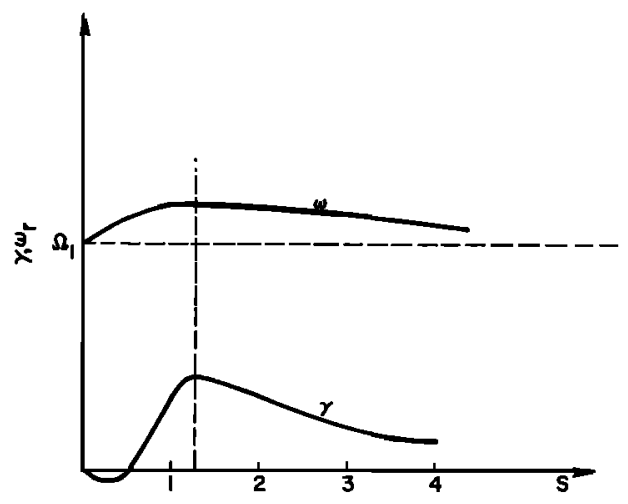

Fig. 7. Qualitative dependence of $\omega_{r}$ and $\gamma$ on wave number $s=$ $k_{1}{ }^{2} V_{i}{ }^{2} / \Omega_{i}{ }^{2}$ for the ion cyclotron instability. 
point the notion of the ponderomotive force becomes important. The ponderomotive force, also known as radiation pressure when the high-frequency fields are light waves, is a nonlinear low-frequency force produced from the coupling of two high-frequency waves. Although this force acts mainly on the electrons, it is ultimately transmitted to the ions by the charge separation field. This force acts as a pressure on the plasma fluid and can thus create density fluctuations. This force is the one that drives the parametric instabilities, which have been
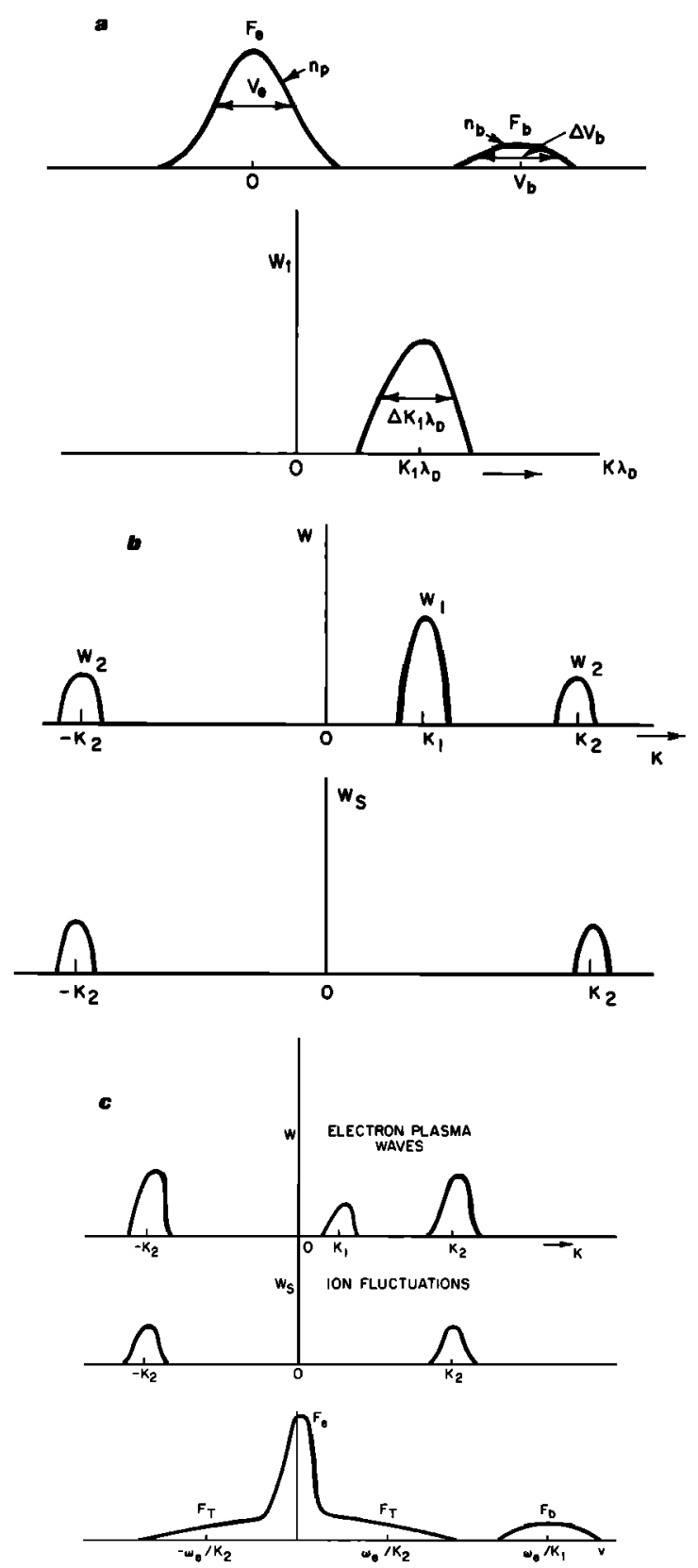

Fig. 8. Pictorial description of the Papadopoulos and Coffey $[1974 a, b]$ model for anomalous resistivity in the aurora. (a) Distribution functions of cold ambient and fast precipitating electrons. They generate primary electron plasma waves $W_{1}$ with wave number $k_{1}=\omega_{e} / V_{b} .(b)$ The primary plasma waves $W_{1}$ parametrically decay to secondary plasma waves $W_{2}$ with longer wavelength $\left(k_{1}<<\right.$ $k_{2}$ ) and nonlinearly modified ion acoustic waves $W_{s} .(c) \mathrm{A}$ quasi-steady state is established, with the precipitated stream nonlinearly stabilized, ambient electron tails $F_{T}, W_{2} \gg W_{1}$, and enhanced level of $W_{s}$. The presence of $W_{s}$ causes anomalous resistivity. the subject of extensive studies with respect to power absorption in laser-produced plasmas: We refer the interested reader to some extensive reviews on this subject written recently [Galeev and Sagdeev, 1973, and references therein]. For our purposes it is sufficient to state that the decay (modified or not) of plasma waves or whistler waves can produce and sustain enhanced density fluctuation spectra which can produce an enhancement in resistivity [Nishihara and Hasegawa, 1972].

The model discussed by Papadopoulos and Coffey [1974a] is then as follows (Figure 8). The precipitating electrons create plasma waves; these waves decay to secondary plasma waves and low-frequency density fluctuations. A quasi-steady state is established according to which the presence of the enhanced density fluctuations stabilizes the primary instability while at the same time provides anomalous resistance to the low-velocity electrons. The details of these mechanisms can be found in work by Papadopoulos and Coffey [1974a, b], Papadopoulos [1975], Matthews et al. [1976], and Papadopoulos and Rowland [1976]. We restrict ourselves here to presenting some of the results found by numerically solving the nonlinear coupled equations discussed by Papadopoulos [1975, equations (2)-(3)] for ionospheric parameters [Palmadesso et al., 1975]. Figure $9 a$ shows the primary waves growing, parametrically exciting the secondary plasma waves and the density fluctuations, and settling to a form of steady state, where the total energy remains constant. Figure $9 b$ shows a typical spectrum of density fluctuations. The details and numerical values of the computation will be published soon. For the purpose of this paper it suffices to state that the order of magnitude of $\nu^{*}$ with $\delta n / n$ $\approx 3 \times 10^{-2}$ (Figure $9 a$ ) and $\overline{k \lambda_{D}} \approx 0.15$ (Figure $9 b$ ) as found from the simulations is

$$
\nu^{*} / \omega_{e} \approx k \lambda_{D}(\delta n / n)^{2} \approx 10^{-s}-10^{-4}
$$

This value is then between 4 and 5 orders of magnitude larger than the collisional value. A detailed paper with respect to the above results is in preparation. There are two additional points that should be made in this section. The first concerns the selfconsistency of the above model. Namely, since, given an anomalous resistivity, runaway electrons can form the beams necessary to produce it, the only requirement is that an electron beam with $V_{b}>10 V_{e}$ exist at some time. The system will then self-consistently produce runaways while the bulk of the electrons see anomalous resistance. We will expand on this in the next section. The second point is that any other highfrequency waves such as whistler or electromagnetic waves can be the cause for the excitations of the density fluctuations and the anomalous resistance. However, at the present time this possibility has not been discussed.

We should mention that the above novel notions on anomalous resistivity are connected with the problem of strong Langmuir turbulence theory, a subject of extremely active research today. Our estimates on resistivity are based on weak plasma turbulence theory as far as the electron-ion wave interaction is concerned. These estimates will be accurate only as long as $\left[1 /\left(\overline{k \lambda_{D}}\right)^{2}\right](\delta n / n)^{2}<1$, which is the condition of validity of weak turbulence theory for the low-frequency modes. Since this is in general obeyed in the ionosphere, we will not proceed to the strong turbulence theory estimates of anomalous resistivity, a rather controversial subject at the present time.

\section{Production of Runaways}

Having reviewed the various mechanisms and the type of anomalous resistivity that they produce, we proceed next to discuss some of their observational consequences. High on this 
list is the problem of electron acceleration. We try to examine below the conditions under which the presence of anomalous resistivity can accelerate particles. At first, it seems peculiar that by increasing the friction force we can accelerate particles. This paradox can be resolved in a similar fashion as in section 3. Namely, one should consider the dependence of the collision frequency on the particle velocity. For Coulomb collisions it was shown to have a $v^{-3}$ dependence. On the basis of this it was concluded that while particles with velocities below $V_{r}$ given by (10) experience large friction, particles with $v>V_{r}$ can be accelerated. These particles are usually called runaways. In determining the runaway processes in the presence of anomalous resistivity the key question concerns the dependence of the anomalous collision frequency on the particle velocity. In a plasma with developed turbulence the fast electrons are acted upon by a force due to the induced Cerenkov effect which leads to quasi-elastic scattering with respect to angles. The collision frequency can be found to be [Rudakov. 1971; Kaplan and Tsytovich, 1973]

$$
\nu^{*}(v)=\nu^{*}\left(V_{e} / v\right)^{3}
$$

where $\nu^{*}$ is the anomalous collision frequency determined for the bulk of the electrons $\left(v \approx V_{e}\right)$ as discussed in section 4 . In a similar fashion as in section 3 we can find the runaway velocity $V_{r}$ by balancing the friction force $-m \nu^{*}(v) v$ against the electric field force $e E$. This leads to $V_{r}=\left(\nu^{*} m V_{e}^{3} / e E\right)^{1 / 2}$. Noting, however, that electron drift velocity is given by $V_{D}=e E / m \nu^{*}$, we find for $V_{D} \lesssim V_{e}$ that

$$
V_{r}=V_{e}\left(V_{e} / V_{D}\right)^{1 / 2}
$$

This is a very important relationship, since it gives the number (or equivalently the flux) of runaways if the drift velocity (or equivalently the cold plasma current) is given. The fraction of particles that run away is

$$
\frac{n_{r}}{n}=\int_{v_{r}}^{\infty} f_{e}\left(v_{\|}\right) d v_{\|}
$$

A very important result can be found with respect to the formation of runaway beams from initially Maxwellian distributions. In this case a rough estimate is given by

$$
\frac{n_{r}}{n}=\exp \left[-\frac{1}{2}\left(\frac{V_{r}}{V_{e}}\right)^{2}\right]=\exp \left[-\frac{1}{2} \frac{V_{e}}{V_{D}}\right]
$$

Notice that (59) and (60) are very general results. They are based only on the dependence given by (57) and are basically independent of the specific anomalous resistivity model.

Equation (60) allows us to draw some general conclusions with respect to the sources of fast precipitating electrons. Instabilities with low thresholds or cold currents with low drift velocity $\left(V_{D} \ll V_{e}\right)$ cannot create any significant percentage of runaways. However, any preexisting number of particles with $v>V_{r}$ will be accelerated. Instabilities with thresholds near $V_{e}$ $\left(V_{D} \approx V_{e}\right)$ like ion acoustic instabilities with $T_{e} \approx T_{l}$, on the other hand, can allow $30-60 \%$ of the particles to run away and therefore can act as strong sources of precipitating particles. Since, as can be inferred from particle flux measurements, large drift velocities are expected to occur at very high altitudes [Kindel and Kennel, 1971; Mozer, 1975], we expect the initial formation of the runaway streams, whether it is due to anomalous resistance or to magnetospheric particle injection, to occur at altitudes higher than $10,000 \mathrm{~km}$. However, once streams with energies larger than 10 times the ambient temper- ature $(\approx 30-100 \mathrm{eV})$ are formed, they can be accelerated continuously till they precipitate. This can occur in a completely self-consistent manner, since as discussed in section 5 , the stream parametrically creates anomalous resistivity which allows for the existence of electric fields much larger than $E_{D}$. These fields can accelerate the fast electrons freely while the force on the bulk of the electrons is balanced by the anomalously high friction. In this model, since the ambient currents can be rather small, the amount of heat generated in situ can be easily transported away.

The above comments apply basically to situations where $V_{D}$ $<V_{e}$. In the opposite case $\left(V_{D}>V_{e}\right),(60)$ predicts that essentially all the electrons are running away. As was noted in section 5 , this was seen in one-dimensional particle simulations despite the fact that Buneman instability developed. The effect of the instability was to transfer half of the available energy into random electron energy, thereby creating hot electron spots. Such spots can of course subsequently create fast electron streams due to thermal expansion. In this way they can provide the initial streams which can subsequently be accelerated. The above statements are valid for the case of homogeneous plasmas (notice that the Buneman type simulations were performed in codes with periodic boundary conditions). When this restriction is removed, the possibility of forming
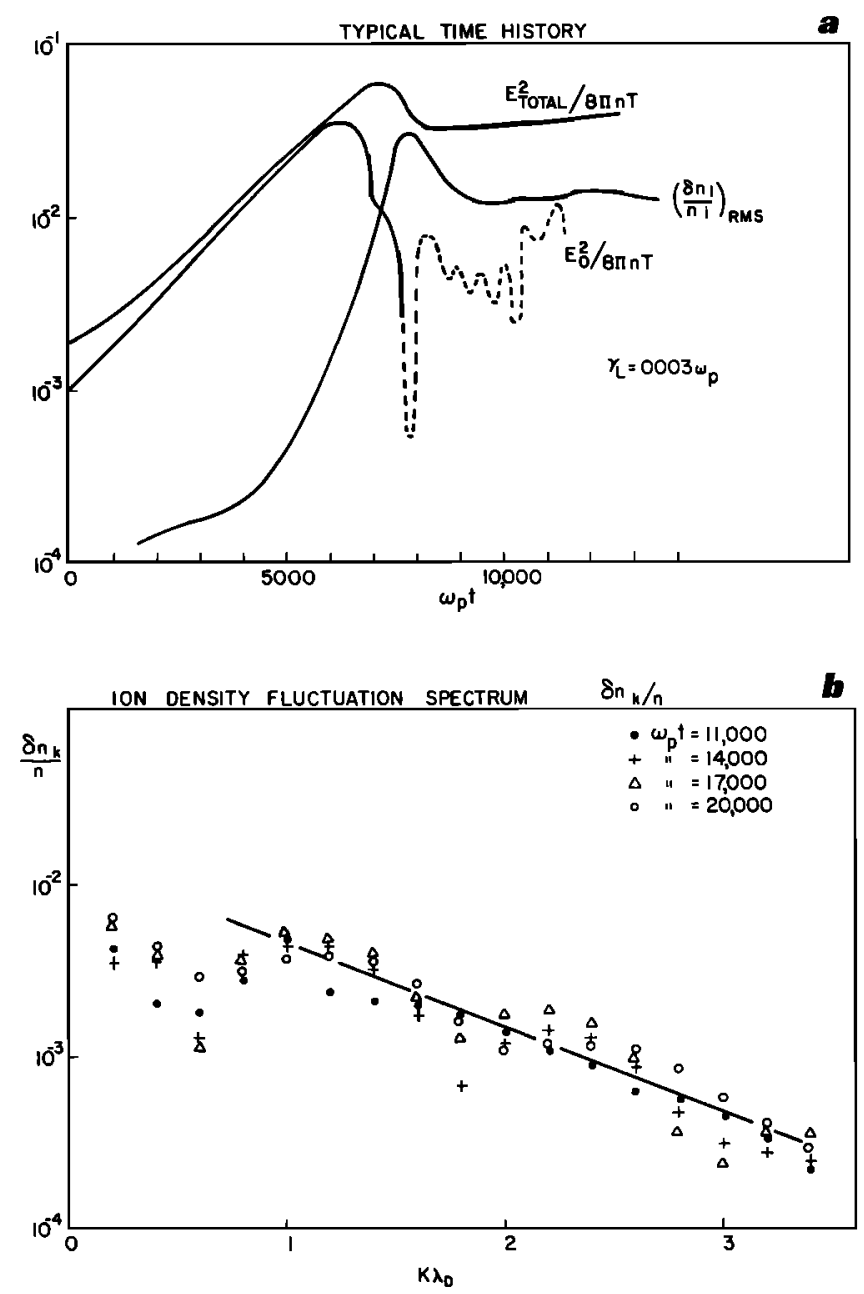

Fig. 9. Results of numerical solution of the nonlinear mode coupled equations for the system of Figure $8 a$ [Palmadesso et al., 1975]. (a) Time evolution of $W_{1}, W_{2}$, and $\delta n / n$, for the case where $\gamma_{b} / \omega_{e}=\left(n_{b} / n\right)\left(V_{b} / \Delta V\right)^{2}$. (b) Stabilized density fluctuation spectrum for the above parameters. 
electrostatic shocks or double layers arises. Since this topic lies outside the scope of the present paper, we refer the interested reader to a recent review by Block [1975] (see also Block [1972]). We only point out here that the necessary condition for double-layer formation is similar to the condition for Buneman instability and therefore very large drifts are required $\left(V_{D} \gg V_{e}\right)$. Again, if the result of the double layer is a formation of a beam, it can subsequently be accelerated in a similar fashion as above. For two-dimensional situations $\left(\omega_{e}>\right.$ $\Omega_{e}$ ) the Buneman instability goes into an ion acoustic instability if the temperature ratio $T_{e} / T_{i}$ allows it.

Before concluding this section we briefly discuss some other observables that result from acceleration in the presence of anomalous resistance. (1) Since the fast electrons are elastically scattered in angle with $\nu(v) \sim 1 / v^{3}$, we expect the more energetic streams to suffer small pitch angle scattering and remain field aligned and nearly monoenergetic. Less energetic streams will tend more toward isotropy. (2) The daughter waves excited parametrically can draw extensive upgoing and downstreaming power law electron tails with energies extending to the energy of the primary stream. (3) The presence of the density fluctuations will inhibit the current independently of its direction and therefore will allow either electric field direction. In this fashion it can lead to ion precipitation, while at the same time it decelerates a previously formed fast electron stream. (4) It can form fast upstreaming and downstreaming ion tails due to the damping of the ion fluctuations in the tails of the ion distribution. The energy of these tails depends on the nonlinear frequency of the ion waves which can be several times the sound speed. A detailed computation of the ion acceleration will be presented elsewhere.

\section{IONOSPHERIC AND MAGNETOSPHERIC OBSERVATIONS AND Theories Related to ANOMAlous Resistivity}

An appropriate presentation of the topic of this section deserves a review article equally extensive to the present one. The importance of connecting the notions presented in the previous sections with the experimental observations and the overall problem of ionospheric-magnetospheric coupling cannot be overemphasized. It was felt, however, that any attempt along this line within the context of the present article would have resulted in making the entire presentation awkward and cumbersome. Nevertheless, for reasons of completeness and for the convenience of the readers we list below some of the main papers covering these subjects without any extensive discussion.

On the experimental side, most of the measurements of lowfrequency ionic waves have been performed in the magnetosphere rather than the ionosphere. Scarf et al. [1973] have reported local detection of field-aligned currents correlated with enhanced electrostatic turbulence at relatively high altitudes on closed $L$ shells on the nightside of the earth. At midaltitudes over the auroral oval [Scarf et al., 1977] they have reported that during moderate magnetospheric disturbances the most intense waves and currents were detected near sharp boundaries in the density of polar cleft electrons. Unfortunately, the interpretation of the observed structures was hindered by the fact that the measurements were performed from a single vehicle rather than a mother-daughter type mission. In addition, Fredricks et al. [1973] and Fredricks and Russell [1973] discussed correlations of wave measurements with magnetometer readings indicating unstable field-aligned currents, and in one particular case they derived an anomalous resistivity of $8 \mathrm{chm} \mathrm{m}$, indicating a potential drop of several kilo- volts between the location of Ogo $5\left(3.2 R_{E}\right)$ and the ionosphere. In the ionosphere, measurements of ion cyclotronlike waves have been reported by Kelley et al. [1975a] at altitudes $400 \mathrm{~km}$ over the auroral zones. Direct measurements of fieldaligned electric fields have been reported by Mozer and Fahleson [1970], Kelley et al. [1975a], and Mozer [1975]. However, it appears that there is a certain amount of skepticism about these measurements due to the significant experimental uncertainties in measuring parallel electric fields. For an extensive reference list on the direct and indirect experimental evidence for anomalous resistivity we refer the interested reader to Mozer [1975].

From the point of view of the importance of anomalous resistivity in the structure of the ionosphere and its coupling to the magnetosphere we mention the significant contributions of Coroniti and Kennel [1972, 1973], Wolf [1975], Sato and Holzer [1973], and more recently the numerical models presented by Fedder [1976]. We should also note the recent work of Evans [1974], who showed that details of some electron energy spectra could be very well explained on the basis of keV-like potential drops between the hot magnetospheric plasma and the ionosphere, and of Kaufmann et al. [1976], who attempted to explain observed auroral spectra by moving the boundaries between which the potential drop occurs.

As mentioned at the beginning, any further commentary on the above topics lies beyond the scope of the present review.

\section{Discussion and Conclusions}

In this review we have remained within the philosophy that essentially exhibits the important physical processes and their consequences as simply as possible. Within that domain we have often had to sacrifice mathematical rigor in the interest of physical understanding. Moreover, we have also often sacrificed detailed and more realistic models of the processes involved so that the physics could more readily be seen. In this spirit we have ignored convective effects due the finite size of the current-carrying regions, an assumption easily justified in almost all situations of interest, with the exception of artificial injection experiments. Thus our main motivation has been to draw forth the basic physical ideas by using the most simplistic situations that we could find.

On the basis of such a framework we have examined the importance of various mechanisms capable of producing anomalous resistivity in the ionospheric plasma and their consequences. These can be broadly subdivided into resistivity produced by instabilities driven by a bulk current and resistivity in a plasma with a stable current.

\section{Current-Driven Instabilities}

It was shown that the existence of current-driven instabilities does not guarantee the appearance of a steady state anomalous resistivity. In addition to a positive answer as to whether the system is linearly unstable to the electron current, one should find the nonlinear limit of the excited waves and then answer the question of whether the anomalous friction and heating rates allow the system to remain in the unstable region. Only if the answer to this question is positive should one accept the linear mechanism as producing anomalous resistivity.

On the basis of the above we pointed out that of the various current-driven instabilities, only the ion acoustic instability when the condition given by (48) is satisfied can account for steady state anomalous resistance. Ion cyclotron and ion acoustic instabilities if the condition of $(48)$ is violated can 
produce only local ion hot spots and transient resistivity spikes. This is because the electron heating rate is smaller than the ion heating rate, and unless some very fast ion cooling process operates the instability, conditions are quickly violated and the waves damp out. It was also shown that instabilities driven by collisions cannot enhance the collision frequency even if $\delta n / n \approx 1$. This is because most of the fluctuation energy is in the form of wave energy rather than field energy, which affects the particle orbits. On the basis of these it was concluded that current-driven instabilities can produce resistivity only at very large altitudes where $V_{D}$ can approach the electron thermal velocity. In addition, under such circumstances a large number of runaways can be produced which can form local electron streams. Such low-energy local streams can also be created by electron heating or double-layer formation at high altitudes, when the conditions for the Buneman instability are satisfied.

\section{Instabilities in a Plasma With a Stable Current}

It was pointed out that independently of whether the plasma is stable or not to current-driven low-frequency instabilities, the existence of high-frequency waves $\left(\omega>\omega_{e}\right)$ due to some nonthermal feature of the high-energy part $\left(v \gg V_{e}\right)$ of the electron distribution function can enhance the bulk plasma resistivity. This occurs when the high-frequency waves can decay efficiently into a pair of another high-frequency wave and a low-frequency wave with phase velocity smaller than $V_{e}$. The presence of the low phase velocity density fluctuations will cause the thermal electrons to see an enhanced friction. A strong candidate for the excitation of the high-frequency waves is the interaction of the auroral streams with ambient plasma. Numerical studies indicate that resistivity can be enhanced by several orders of magnitude. The model emerging is that an initial runaway streamer of modest energy $\left(\sim 10-20 T_{e}\right)$ can be accelerated to energies of $10-30 \mathrm{keV}$ while self-consistently producing the necessary resistivity that allows for the existence of the potential drop. At the same time it can produce by collisionless processes secondary power law tails similar to those observed [Reasoner and Chappell, 1973; Matthews et al., 1976].

In concluding, we wish to point out that the situations considered here by no means encompass all aspects of the problem. They represent what we believe to be the current state of the art, and as such they unavoidably represent our own biases. What we believe is needed next is comprehensive numerical study in which the theories discussed above will be included in a realistic model with gradients, boundary conditions, and multispecies ions in a fashion similar to the numerical models of Fedder [1976]. Such a program should be guided and coordinated by in situ measurements of density fluctuation spectra correlated with measurements of field-aligned currents, energy spectra of energetic electrons, and if possible parallel electric fields. The importance of carrying similar measurements with a mother-daughter mission cannot be overestimated. We believe that this type of combined experimental and theoretical study properly coordinated from the planning stage till its completion can contribute significantly toward the resolution of the important problems of the auroral plasma.

\section{NOTATION}

$n$ number density of ambient plasma.

$n_{b}$ number density of precipitating electrons.

$n_{r}$ number density of runaway electrons. $\delta$ density fluctuation.

$\checkmark$ electron velocity.

$V_{D}$ relative drift between electrons and ions.

$V$, thermal velocity of species $j$.

$V_{r}$ runaway velocity.

$V_{\text {cr }}$ critical velocity for ion acoustic and Buneman instabilities.

$V_{b}$ velocity of precipitating electrons.

$\Delta V_{b}$ velocity spread of precipitating electrons.

$v_{\mathrm{ph}}$ phase velocity, equal to $\omega_{r} / k$.

$C_{s}$ sound speed.

$e$ electron charge.

$q_{j}$ charge for species $j$.

$T$, temperature for species $j$.

$m(M)$ electron (ion) mass.

$j$ current density.

E electric field.

$\mathbf{E}_{D}$ Dreicer or runaway field.

$\omega_{j}$ plasma frequency for species $j$.

$\Omega$ cyclotron frequency for species $j$.

$\omega$ complex frequency.

k wave number.

$\omega_{r}\left(\omega_{k}\right)=\operatorname{Re} \omega\left(\operatorname{Re} \omega_{k}\right)$.

$\gamma\left(\gamma_{k}\right)=\operatorname{Im} \omega\left(\operatorname{Im} \omega_{k}\right)$.

( $)_{i}$ ion (proton).

( )e electron.

( ) parallel to the magnetic field.

( ) $)_{\perp}$ perpendicular to the magnetic field.

( ) quantity evaluated with linear orbits in a stable system.

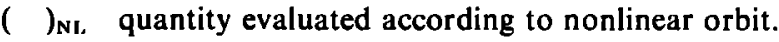

$\eta$ resistivity.

$\sigma$ conductivity.

$\nu$ binary collision frequency.

$\nu^{*}$ anomalous collision frequency.

$\nu^{*}(v)$ velocity dependent collision frequency due to collective effects in a stable plasma.

$\lambda_{D}$ electron Debye length.

$\Lambda$ number of particles in a Debye sphere, equal to $4 \pi n \lambda_{D}{ }^{3}$.

$F$ friction force.

$B$ magnetic field.

E plasma dielectric constant.

$\chi_{j}$ polarizability for species $j$.

$\left.\left\langle E^{2}\right|\right)$ electric field fluctuation spectrum.

$S_{i j}($ ) density correlation function between species $i$ and $j$.

$W$ total electric field energy density, equal to $\left\langle E^{2}\right\rangle / 8 \pi$.

Acknowledgments. It is impossible to acknowledge the contribution of all the people whose ideas contributed to this paper. Specific thanks go to T. Coffey, P. Palmadesso, S. Ossakow, and J. Fedder. The expert numerical help of $I$. Haber is very much appreciated. I also thank F. Scarf for useful suggestions on the manuscript. Work was supported by the Office of Naval Research.

\section{REFERENCES}

Benford, G., Electrostatic ion cyclotron waves: Plasma heating, frequency shifts and line widths, $J$. Plasma Phys., in press, 1976.

Biskamp, D., On conventional and unconventional theory of ion sound turbulence, Nucl. Fusion, 12, 85, 1972.

Biskamp, D., and R. Chodura, Asymptotic behavior of the twostream instability, Phys. Fluids, 16, 888, 1973.

Block, L. P., Potential double layers in the ionosphere, Cosmic Electrodynamics, 3, 349, 1972.

Block, L. P., Double layers, in Physics of the Hot Plasma in the Magnetosphere, p. 229, Plenum, New York, 1975. 
Boris, J. P., J. M. Dawson, J. H. Orens, and K. V. Roberts, Computations of anomalous resistance, Phys. Rev. Lett., 25, 706, 1970.

Buneman, O., Dissipation of currents in ionized media, Phys. Rev., $115,503,1959$.

Caponi, M.Z., and R. C. Davidson, Influence of ion tail formation on the dynamic behavior of a current carrying plasma, Phys. Fluids, 17, 1394, 1974.

Choi, D., and W. Horton, Modified Kadomtsev spectrum from renormalized plasma theory, Phys. Fluids, 17, 2048, 1974.

Coppi, B., and E. Mazzucato, Anomalous resistivity in low electric fields, Phys. Fluids, 14, 134, 1971.

Coroniti, F. V., and C. F. Kennel, Polarization of the auroral electrojet, J. Geophys. Res., 77, 2835, 1972.

Coroniti, F. V., and C. F. Kennel, Can the ionosphere regulate magnetospheric convection?, J. Geophys. Res., 78, 2837, 1973.

Davidson, R. C., N. A. Krall, K. Papadopoulos, and R. Shanny, Electron heating by electron-ion stream instabilities, Phys. Rev. Lett., 24, 579, 1970.

Drummond, W. E., and M. N. Rosenbluth, Anomalous diflusion arising from microinstabilities in a plasma, Phys. Fluids, 5, 1507, 1962.

Dum, C. T., and T. D. Dupree, Non-linear stabilization of high frequency instabilities in a magnetic field, Phys. Fluids, 13, 2064, 1970.

Dupree, T. H., A perturbation theory for strong plasma turbulence, Phys. Fluids, 9, 1773, 1966.

Dupree, T. H., Theory of resistivity in collisionless plasma, Phys. Rev. Lett., 25, 789, 1970.

Evans, D. S., Precipitating electron fluxes formed by a magnetic field aligned potential difference, J. Geophys. Res., 79, 2853, 1974.

Fedder, J. A., Effect of anomalous resistivity on auroral Birkeland current systems, Ann. Geophs., in press, 1976.

Fredricks, R. W., and C. T. Russell, Ion cyclotron waves observed in the polar cusp, J. Geophys. Res., 78, 2917, 1973.

Fredricks, R. W., F. L. Scarf, and C. T. Russell, Field-aligned currents, plasma waves, and anomalous resistivity in the disturbed polar cusp, J. Geophys. Res., 78, 2133, 1973.

Galeev, A. A., and R. Z. Sagdeev, Parametric phenomena in a plasma, Nucl. Fusion, 13, 603, 1973.

Gary, S. P., and J. W. M. Paul, Anomalous resistivity due to electrostatic turbulence, Phys. Rev. Lett., 26, 1097, 1971.

Hasegawa, A., Instabilities and nonlinear processes in geophysics and astrophysics, Rev. Geophys. Space Phys., 12, 273, 1974.

Hasegawa, A., Plasma Instabilities and Non-Linear Effects, chap. 2, Springer, New York, 1975.

Hirshfield, J. L., Wave-wave contribution to the high frequency resistivity of nonequilibrium plasma, Phys. Fluids, 16, 1460, 1973.

Horton, W., D. Choi, and R. A. Koch, Ion acoustic heating from renormalized turbulence theory, Phys. Rev.. Sect. A, 14, 424, 1976.

Hudson, M. K., Additional free energy sources for anomalous resistivity to field aligned currents, paper presented at 16th IUGG General Assembly, Grenoble, France, 1975.

Hui, B. H., and T. H Dupree, Computer experiments on clump formation in plasma, Phys. Fluids, 18, 235, 1975.

Ichimaru, S., Basic Principles of Plasma Physics, chap. 3 and 9, W. A. Benjamin, Reading, Mass., 1973.

Jackson, E. A., Drift instabilities in a Maxwellian plasma, Phys. Fluids, 3, 786, 1960.

Kadomtsev, B. B., Plasma Turbulence, chap. 2 and 4, Academic, New York, 1965.

Kaplan, S. A., and V. N. Tsytovich, Plasma Astrophysics, p. 134, Pergamon, New York, 1973.

Karatzas, N., A. Anastassiadis, and K. Papadopoulos, Generation and behavior of large amplitude ion acoustic waves, Phys. Rev. Lett., 35, 33, 1975.

Kaufmann, R. L., D. N. Walker, and R. L. Arnoldy, Acceleration of auroral electrons in parallel electric fields, J. Geophys. Res., 81, 1673, 1976.

Kelley, M. C., G. Haerendel, H. Kappler, F. S. Mozer, and U. V. Fahleson, Ionospheric fields in a substorm, J. Geophys. Res., 80. $3181,1975 a$.

Kelley, M. C., E. A. Bering, and F. S. Mozer, Evidence that the electrostatic ion cyclotron instability is saturated by ion heating, Phys. Fluids, 18, 1590, 1975 b.

Kindel, J. M., and C. F. Kennel, Topside current instabilities, J. Geophys. Res., 76, 3055, 1971.

Lampe, M., I. Haber, J. H. Orens, and J. P. Boris, Two dimensional study of electron ion streaming instabilities, Phys. Fluids, 17, 428, 1974.

Manheimer, W. M., and R. W. Flynn, Formation of non-thermal ion tails in the ion acoustic instability, Phys. Fluids, 17, 409, 1974.

Matthews, D. L., M. Pongratz, and K. Papadopoulos, Nonlinear production of suprathermal tails in auroral electrons, J. Geophys. Res., 81, 123, 1976.

McBride, J. B., and T. K. Chu, Ion acoustic instability of a collisional plasma with nonstationary equilibrium, Phys. Fluids, 15, 1682, 1972.

Mikhailovskii, A. B., Theory of Plasma Instabilites, vol. 1, chap. 3 and 4, Consultants Bureau, New York, 1974.

Montgomery, D. C., and D. A. Tidman, Plasma Kinetic Theory, chap. 5 and 8, McGraw-Hill, New York, 1964.

Morse, R. L., and C. W. Nielson, Studies of turbulent heating of hydrogen plasmas by numerical simulation, Phys. Rev. Lett. 26. 3, 1971.

Mozer, F. S., Anomalous resistivity and parallel electric fields, technical report, Space Sci. Lab., Univ. of Calif., Berkeley, 1975.

Mozer, F. S., and U. V. Fahleson, Parallel and perpendicular electric fields in the auroral ionosphere, Planet. Space Sci., 18, 1563, 1970.

Nishihara, K., and A. Hasegawa, Effect of an external circuit on anomalous resistivity in plasmas, Phys. Rev. Lett., 28, 424, 1972.

Orens, J. H., and J. M. Dawson, Anomalous D. C. plasma resistivity due to ion acoustic lurbulence, Memo Rep. 2850, Nav. Res. Lab., Washington, D. C., 1974.

Ossakow, S. L., Anomalous resistivity along lines of force in the magnetosphere, J. Geophys. Res., 73, 6366, 1968.

Palmadesso, P. J., T. P. Coffey, S. L. Ossakow, and K. Papadopoulos, Topside ionosphere ion heating due to electrostatic ion cyclotron turbulence, Geophys. Res. Lett., I, 105, 1974.

Palmadesso, P. J., T. P. Coffey, I. Haber, and K. Papadopoulos, Parametric stabilization of relativistic and non-relativistic beam plasma instabilities, Bull. Amer. Phys. Soc., 20, 1277, 1975.

Papadopoulos, K., Evaluation of the wave energy of a plasma approaching the unstable ion acoustic region, M.Sc. thesis, Dep. of Nucl. Eng., Mass. Inst. of Technol., Cambridge, 1965.

Papadopoulos, K., Bremsstrahlung radiation in plasmas, Amer. $J$. Phys., 38, 87, 1970.

Papadopoulos, K., Non-linear stabilization of beam plasma interactions by parametric effects, Phys. Fluids, 18, 1769, 1975.

Papadopoulos, K., and T. Coffey, Nonthermal features of the auroral plasma due to precipitating electrons, J. Geophys. Res., 79, 674, $1974 a$.

Papadopoulos, K., and T. Coffey, Anomalous resistivity of the auroral plasma, J. Geophys. Res., 79, 1558, 19746.

Papadopoulos, $K$., and $H$. Rowland, Collisionless effects on the spectrum of secondary electrons at low altitudes, submitted to $J$. Geophys. Res., 1976.

Petviashvili, V. I., Non-linear oscillations and some effects due to a longitudinal current in a plasma, Zh. Eksp. Teor. Fiz., 45, 1467, 1963. (Sov. Phys. JETP, Engl. Transl., 18, 1014, 1964.)

Reasoner, D. L., and C. R. Chappell, Twin pay load observations of incident and backscattered auroral electrons, J. Geophys. Res., 78, 2176, 1973.

Ronglien, T. D., and S. A. Self, Ion acoustic instability of a collisional, fully ionized plasma, Phys. Rev. Lett., 27, 792, 1971.

Rostoker, N., Fluctuations in a plasma, Nucl. Fusion, l, 101, 1961.

Rudakov, L. I., Nature of turbulent heating in the tokomak TM-3 device, Zh. Eksp. Teor. Fiz., 60, 2132, 1971. (Sov. Phys. JETP, 33, 1148, 1971.)

Sagdeev, R. Z., Advances in Plasma Physics, edited by A. Simon and W. B. Thomson, vol. 5, p. 153, John Wiley, New York, 1974.

Sagdeev, R. Z., and A. A. Galeev, Nonlinear Plasma Theory, pp. 54, 103, W. A. Benjamin, New York, 1969.

Sato, T., and T. E. Holzer, Quiet auroral arcs and electrodynamic coupling between the ionsophere and the magnetosphere, $J$. Geophys. Res., 78, 7314, 1973.

Scarf, F. L., et al., Observation of a current-driven plasma instability at the outer zone-plasma sheet boundary, J. Geophys. Res., 78, $2150,1973$.

Scarf, F. L., et al., Current-driven plasma instabilities at high latitudes, J. Geophys. Res., 82, in press, 1977.

Self, S. A., Ion waves, drift waves and instability at a weakly ionized magnetoplasma, J. Plasma Phys., 4, 693, 1970.

Sitenko, A. G., Electromagnetic Fluctuations in a Plasma, chap. 5, Academic, New York, 1967.

Sleeper, A. M., and J. Weinstock, Non-linear theory of density fluctuations in turbulent plasmas, Phys. Fluids, 15, 1507, 1972. 
Sleeper, A. M., J. Weinstock, and B. Bezzerides, Non-linear theory and angular spectrum of the ion acoustic instability, Phys. Fluids, 16, 1508, 1973.

Spitzer, L., Physics of Fully Ionized Gases, chap. 5, Interscience, New York, 1956.

Tange, T., and S. Ichimaru. Theory of anomalous resistivity and turbulent heating in plasmas, J. Phys. Soc. Jap., 36. 1437, 1974.

Tsytovich, V. N., The spectra and correlation functions for ion sound turbulence, Plasma Phys., 13, 741, 1971.

Tsytovich, V. N., An Introduction to the Theory of Plasma Turbulence, chap. 4 and 5, Pergamon, New York, 1972.
Vedenov, A. A., Theory of Turbulent Plasma, pp. 66-67, Elsevier, New York, 1968.

Weinstock, J., Kinetic equation to higher orders in the plasma parameters, Phys. Fluids, 10, 127, 1967.

Wesson, J. A., and A. Sykes, Theory of ion sound resistivity, Phys. Rev. Lett., 3I, 449, 1973.

Wolf, R. A., Ionosphere-magnetosphere coupling, Space Sci. Rev., 17. $537,1975$.

(Received April 5, 1976; accepted September 16, 1976.) 